\title{
DIFFERENTIABLE MONOTONE MAPS ON MANIFOLDS
}

\author{
BY \\ P. T. CHURCH( $\left.{ }^{1}\right)$
}

1. Introduction. If $X$ and $Y$ are separable metric spaces and $f: X \rightarrow Y$ is a map, the branch set $B_{f}$ is the set of points at which $f$ fails to be a local homeomorphism. The map $f$ is called proper if for each compact set $W \subset Y, f^{-1}(W)$ is compact; in particular, if $X$ is compact then $f$ is proper. A proper map $f: X \rightarrow Y$ is called monotone if for each $y \in Y, f^{-1}(y)$ is connected (it may be empty). Most authors require that a monotone map be onto, but we consider the more general situation (only) in (2.1) and (2.2).

Part of the interest in monotone maps is due to the monotone-light factorization theorem [32, pp. 141-142] proved independently by Eilenberg and Whyburn: If $f: X \rightarrow Y$ is proper and $Y$ is locally compact, then there exists a unique factorization $f=h g$, where $g$ is monotone (onto) and $h$ is light (i.e., for each $y \in Y$, $\operatorname{dim} f^{-1}(y) \leqq 0$ ). If $X$ and $Y$ are differentiable $n$-manifolds, and $f: X \rightarrow Y$ is differentiable, a natural question thus arises-under what conditions can $g$ and $h$ also be chosen to be differentiable? If $f$ is $C^{3}$, then one condition is that $\operatorname{dim}\left(B_{f}\right) \leqq n-3$; in fact, $h$ is a diffeo-covering map in this case [8]. In an effort to answer this question more generally, and to characterize the map $g$ of [8] more fully, it seems worthwhile to study the differentiable monotone maps $f: M^{n} \rightarrow N^{n}$ on $n$-manifolds (without boundary).

A contractible, compact $n$-manifold with simply-connected boundary is called a homotopy $n$-cell. A compact subset $A$ of an $n$-manifold $M^{n}$ is acyclic if it has the integral Čech cohomology groups of a point; $A$ is homotopy cellular if there exist homotopy $n$-cells $A_{k} \subset M^{n}$ such that $\bigcap_{k} A_{k}=A$ and $A_{k+1} \subset$ int $\left(A_{k}\right)$; it is cellular [3] if, in addition, each $A_{k}$ is an $n$-cell. If $M^{n}$ and $N^{n}$ are $n$-manifolds without boundary, a proper map $f: M^{n} \rightarrow N^{n}$ is acyclic (resp., homotopy cellular, cellular) if, for each $y \in N^{n}, f^{-1}(y)$ is acyclic (resp., homotopy cellular, cellular).

Standing hypothesis. Whenever the statement of a theorem refers to a $C^{m}$ map $f$ without specifying its domain and range, it is understood that $f: M^{n} \rightarrow N^{n}$ is proper, where $M^{n}$ and $N^{n}$ are $C^{m}$ connected (separable) n-manifolds without boundary $(m=0,1, \ldots)$.

The main theorem of this paper, proved in (4.4) and (4.6), is:

Presented to the Society, January 29, 1965; received by the editors September 14, 1964 and, in revised form, June 10, 1966.

( ${ }^{1}$ Research supported by National Science Foundation grant GP-2193; revision supported by an NSF Senior Postdoctoral Fellowship at the Institute for Advanced Study. 
1.1. TheOREM. (a) If $f$ is $C^{n}$ monotone and $\operatorname{dim}\left(B_{f}\right)<n / 2$, or

(b) if $f$ is $C^{n}$ acyclic and $\operatorname{dim}\left(B_{f}\right) \leqq n-2$,

then $f$ is homotopy cellular; if $n \neq 3,4$, or 5 , then $f$ is cellular.

In fact the sets $A_{k}$ may be chosen to be $C^{n}$ manifolds. Examples are given ((2.5), (2.12), (2.14), and (4.5)) to show that the hypotheses on $\operatorname{dim}\left(B_{f}\right)$ are required. The simplicial analog is trivially true (4.8).

The factorization theorem of [8] thus implies:

1.2. CoRollary. If $f$ is $C^{m}(m \geqq n \geqq 4)$ and $\operatorname{dim}\left(B_{f}\right)<n / 2$, then there exists a unique factorization $f=h g$, where $g: M^{n} \rightarrow K^{n}$ is a $C^{m}$ homotopy cellular map (cellular if $n \neq 4,5$ ), $K^{n}$ is a $C^{m}$ manifold, and $h: K^{n} \rightarrow N^{n}$ is a $C^{m}$ diffeo-covering map.

For $n=1,2$, and $3(1.2)$ is easily seen to be false.

The results in this paper all deal directly with differentiable monotone or quasimonotone maps (§2), with two exceptions. In $\S 5$ a question of Hopf about essential maps of $S^{3}$ into $S^{2}$ is answered (the monotone map involved is, of course, the Hopf fibering). And a theorem on quasi-monotone maps (2.6) leads naturally to (1.3), stated below and proved in (2.10).

If $f: M^{m} \rightarrow N^{n}$ is $C^{1}$, let $R_{k}(f)$ be the set of points at which the Jacobian matrix (derivative map) of $f$ has rank at most $k$. If $f$ is $C^{m-k}$, then [25, p. 173, Theorem 2] $\operatorname{dim}\left(f\left(R_{k}(f)\right)\right) \leqq k$; if $f$ is proper, then $f\left(R_{k}(f)\right)$ is closed in $N^{n}$.

1.3. THEOREM. If $f$ is $C^{2}$ and $M^{n}$ and $N^{n}$ are oriented, then for each $y \in N^{n}$ $-f\left(R_{n-2}(f)\right), f^{-1}(y)$ has at least $|\operatorname{deg} f|$ components.

See (1.6) below; [28, p. 128, Theorem (4.3)] can be viewed as a consequence of (1.3). The complex analytic function $f: S^{2} \rightarrow S^{2}$ defined by $f(z)=z^{2}$ shows that $f\left(R_{n-2}(f)\right)$ cannot be replaced by a subset of smaller dimension.

1.4 Conventions. Throughout this paper manifolds are separable and without boundary, unless otherwise specified. The tangent bundle of $M^{n}$ is denoted by $T M^{n}$, and the tangent space at $x \in M^{n}$ by $T_{x} M^{n}$. Coordinates are written up $x^{i}$, a map is a continuous function, and the composition of two functions is denoted by $g f$ or $g \circ f$.

Čech homology and cohomology are consistently used, $Z$ (resp. $Z_{p}$ ) is the group of integers (integers $\bmod p$ ), and the (weak) direct sum is denoted by $\Sigma$. The boundary of a set $X$ is denoted by bdy $X$ or $\partial X$ (in case $X$ is a manifold with boundary), the interior of $X$ by int $X$, the closure of $X$ by $\bar{X}$ or $\mathrm{Cl}[X]$, and the restriction of the map $f$ to $X$ by $f \mid X$. The distance between two points is $d(x, y)$, and $S(x, \varepsilon)=\{y: d(x, y)<\varepsilon\}$. The $n$-sphere is denoted by $S^{n}$, euclidean $n$-space by $E^{n}$, the origin vector in $E^{n}$ by 0 , and the closed ball $\mathrm{Cl}[S(0,1)]$ in $E^{n}$ by $D^{n}$.

1.5. REMARK. Except in $\S 5$ each theorem deals with $C^{m}(m \geqq 1)$ manifolds and a $C^{m}$ map $f: M^{n} \rightarrow N^{n}$. Since each property of hypothesis and conclusion is invariant under $C^{m}$ diffeomorphisms, we may as well suppose that each of $M^{n}$ 
and $N^{n}$ is a $C^{\infty}[22$, p. 41] complete Riemannian manifold [22, p. 20] with induced triangulation $[22$, p. 101, (10.6)] and distance function induced by its Riemannian metric $[17$, p. $166,(3.5)]$.

Furthermore the differentiability hypotheses in Thom's Transversality Lemma $[29$, p. 26] can be improved $[8$, p. $376,(2.6)]$. See also $[8$, p. $376,(2.5)]$.

1.6. Oriented $C^{m}$ manifolds are defined in $[28$, p. $115,(3.7)]$ and characterized in [28, p. 116, Theorem 3.3]. For connected oriented $C^{m}$ manifolds $M^{n}$ and $N^{n}$, and a proper $C^{m}$ map $f: M^{n} \rightarrow N^{n}$, the degree $\operatorname{deg}(f)$ is defined and characterized in [28, p. 127, Theorem 4.2]. In particular, it is meaningful [28, p. 127] to talk of the sign of the Jacobian determinant $J$ at a point $x$, i.e., $J(x)>0,=0$, or $<0$.

2. General properties of monotone and quasi-monotone maps. If $X$ is a locally connected generalized metric continuum and $Y$ is a separable metric space, a proper map $f: X \rightarrow Y$ is called quasi-monotone [32, p. 152] if and only if, for each region $U \subset Y$ and component $V$ of $f^{-1}(U), f(V)=U$. Both monotone onto maps and proper open maps are quasi-monotone, and conversely, any quasi-monotone map $f$ can be (uniquely) factored $f=h g$, where $g$ is monotone (onto) and $h$ is light open [32, pp. 151-155]. On locally connected continua the quasi-open maps of [26, p. 110] are quasi-monotone, and [34] is devoted to a study of these maps in case $X$ and $Y$ are 2-manifolds. Some results on differentiable quasi-monotone maps are given in [6], [30], and [31].

A $C^{2}$ proper map $f: M^{n} \rightarrow N^{n}$ with $\operatorname{dim}\left(B_{f}\right) \leqq n-2$ is quasi-monotone [6, p. 380, (3.2)]. More generally, if $\operatorname{dim} B_{f} \leqq n-1$ and the Jacobian $J \geqq 0$ or $J \leqq 0$ locally at each point of $M^{n}$, then $[6,(3)] f$ is quasi-monotone.

2.1. Lemma. Let $f$ be $C^{2}$, let $n \geqq 2$, and let $y(i)$ be distinct points in $N^{n}-f\left(R_{n-2}(f)\right)$ $(i=1,2)$. Then

(a) Each component of $f^{-1}(y(i))$ is a point, or a $C^{2}$ embedding of a closed interval or $S^{1}$.

(b) If $f$ is monotone or quasi-monotone, then $f^{-1}(y(i))$ has a finite number of components.

(c) If $f^{-1}(y(i))$ has a finite number of components, then there are a $C^{2}$ diffeomorphism $\rho$ of an open subset of $N^{n}$ onto $S^{1} \times E^{n-1}$, and a $C^{2}$ diffeomorphism $\sigma$ of $L^{1} \times E^{n-1}$ onto $f^{-1}\left(\rho^{-1}\left(S^{1} \times E^{n-1}\right)\right)$, where $L^{1}$ is the disjoint union of copies of $S^{1}, \rho f \sigma\left(S^{1} \times\{t\}\right) \subset S^{1} \times\{t\}$, and $\rho(y(i)) \in S^{1} \times\{0\}\left(i=1,2 ; t \in E^{n-1}\right)$.

Proof. We may suppose (1.5) that $M^{n}$ and $N^{n}$ are $C^{\infty}$ manifolds. For each $\bar{x} \in f^{-1}(y(i))$, there are [7, p. 87, (1.1)] $C^{2}$ diffeomorphisms $\lambda$ of a neighborhood $U(\bar{x})$ of $\bar{x}$ onto $E^{n}$ and $\mu$ of a neighborhood $V(y(i))$ of $y(i)$ onto $E^{n}$ such that the map $g=\mu f \lambda^{-1}$ has $g^{j}\left(x^{1}, x^{2}, \ldots, x^{n}\right)=x^{j}(j=1,2, \ldots, \mathrm{n}-1)$; conclusion (a) follows.

If $f$ is either monotone or quasi-monotone, then for each $\left(x^{1}, x^{2}, \ldots, x^{n-1}\right) \in E^{n-1}$ the map $h: E^{1} \rightarrow E^{1}$ defined by $g^{n}\left(x^{1}, x^{2}, \ldots, x^{n}\right)=h\left(x^{n}\right)$ is monotone. Since $f$ is proper, conclusion (b) follows.

For (c) let $\Omega_{i, j}$ be the components of $f^{-1}(y(i))\left(j=1,2, \ldots, k_{i} ; i=1,2\right)$. If 
$\Omega_{i, j}=\{x\}$ where $x \notin R_{n-1}(f)$, let $\Gamma_{i, j}$ be any $(n-1)$-subspace of the tangent space $T_{y(i)} M^{n}$; otherwise, by the argument of the second and third paragraphs of $[8$, p. $378,(3.1)]$ there is a unique $(\mathrm{n}-1)$-subspace $\Gamma_{i, j} \subset T_{y(i)} M^{n}$ such that $f_{*}\left(T_{x} M^{n}\right)$ $=\Gamma_{i, j}$ for each $x \in \Omega_{i, j}$.

There is a $C^{\infty}$ diffeomorph $\Lambda \subset N^{n}$ of $E^{1}$ with $y(i) \in \Lambda$; thus [24] there is a $C^{\infty}$ diffeomorph $U \subset N^{n}$ of $E^{n}$ with $y(i) \in U(i=1,2)$. Let $\alpha: S^{1} \rightarrow U$ be a $C^{\infty}$ embedding such that $y(i) \in \alpha\left(S^{1}\right), \alpha\left(S^{1}\right)$ has trivial normal bundle, and $T_{y(i)} \alpha\left(S^{1}\right)$ is transverse to $\Gamma_{i, j}\left(j=1,2, \ldots, k_{i} ; i=1,2\right)$. Let $V$ be a tubular neighborhood of $\alpha\left(S^{1}\right)-\{y(1), y(2)\}$ in $U-\{y(1), y(2)\}$; by [29, p. 26] there is a $C^{2}$ diffeomorphism $A$ of $N^{n}$ onto itself such that $A$ is the identity map off $V$ and $f$ is transverse regular [29, p. 23] on $A^{-1}\left(\alpha\left(S^{1}\right)-\{y(1), y(2)\}\right)$; thus $f$ is transverse regular on $A^{-1}\left(\alpha\left(S^{1}\right)\right)$.

Let $\rho$ be a $C^{2}$ diffeomorphism of a tubular neighborhood of $A^{-1}\left(\alpha\left(S^{1}\right)\right)$ onto $S^{1} \times E^{n-1}$ with $\rho\left(A^{-1}\left(\alpha\left(S^{1}\right)\right)\right)=S^{1} \times\{0\}$. Let $\varepsilon>0$ and $\sigma$ be as given by [8, p. $376,(2.7)]$; then each component of $L^{1}=f^{-1}\left(A^{-1}\left(\alpha\left(S^{1}\right)\right)\right)$ is $\left(C^{2}\right.$ diffeomorphic to $)$ $S^{1}$. We may as well suppose that $S(0, \varepsilon)=E^{n-1}$, and (c) follows.

\subsection{TheOREM. Let $f$ be $C^{2}$ monotone.}

(1) If $f$ is onto, then (i) for each $y \in N^{n}, f^{-1}(y)$ does not separate $M^{n}$ if $n \geqq 2$, and (ii) for each $y \in N^{n}-f\left(R_{n-2}(f)\right), f^{-1}(y)$ is a point or a $C^{2}$ embedding of $[0,1]$.

(2) If $f$ is not onto, then (i) $B_{f}=R_{n-1}(f)=M^{n}$ (so that $\operatorname{dim}\left(f\left(M^{n}\right)\right) \leqq n-1$ [28, p. 47, Theorem 3.1]), and (ii) $f^{-1}\left(N^{n}-f\left(R_{n-2}(f)\right)\right)$ is the space of a fiber bundle over a (not necessarily connected) (n-1)-manifold with fiber $S^{1}$ and projection $f$.

Proof. If $n=1$ and $f$ is onto, then conclusion (1)(ii) is satisfied; if $f$ is not onto, then $M^{1}=S^{1}$ and $f$ is constant. Thus we may suppose than $n \geqq 2$.

Let $A_{1}$ (respectively, $\left.A_{2}\right)$ be the subset of $N^{n}-f\left(R_{n-2}(f)\right)$ consisting of points $y$ with $f^{-1}(y)$ a point or a $C^{2}$ embedding of a closed interval (resp., the empty set or a $C^{2}$ embedding of $\left.S^{1}\right)$. By (2.1)(a) $A_{1} \cup A_{2}=N^{n}-f\left(R_{n-2}(f)\right)$. For each $y \in f\left(M^{n}\right)$ $-f\left(R_{n-2}(f)\right)$, there is a point $y_{1} \neq y, y_{1} \in N^{n}-f\left(R_{n-1}(f)\right)$ [28, p. 47, Theorem 3.1]; let $\rho$ and $\sigma$ be as given by (2.1)(b) and (c) for $y$ and $y_{1}$.

For $y \in A_{1}$ and $S^{1}$ the component of $L^{1}$ containing $f^{-1}(y)$, the restriction map

$$
\rho f \sigma \mid\left(S^{1} \times\{0\}\right): S^{1} \times\{0\} \rightarrow S^{1} \times\{0\}
$$

is not constant, and since it is monotone, it is thus essential. Thus for each $t \in S(0, \varepsilon)$ the map $\rho f \sigma \mid\left(S^{1} \times\{t\}\right)$ is essential, and hence onto. Since $f$ is monotone, $L^{1}=S^{1}$; moreover, for each $u \in S^{1} \times S(0, \varepsilon),(\rho f \sigma)^{-1}(u)$ is not homeomorphic to $S^{1}$. As a result $A_{1}$ is open.

For $y \in A_{2} \cap f\left(M^{n}\right)$ and $S^{1}$ a component of $L^{1}$, the map $\rho f \sigma \mid\left(S^{1} \times\{0\}\right)$ is constant, and it follows as above that $\rho f \sigma \mid\left(S^{1} \times\{t\}\right)$ is constant for each $t \in S(0, \varepsilon)$. Thus $A_{2}$ is also open. Since $f$ is proper, $f\left(R_{n-2}(f)\right)$ is closed; $\operatorname{since} \operatorname{dim}\left(f\left(R_{n-2}(f)\right)\right)$ $\leqq n-2\left[25\right.$, p. 173, Theorem 2], $N^{n}-f\left(R_{n-2}(f)\right)$ is connected. Thus either $A_{1}=\varnothing$ or $A_{2}=\varnothing$. 
Suppose $A_{2}=\varnothing$. Then $N^{n}-f\left(R_{n-2}(f)\right) \subset f\left(M^{n}\right)$, and, since $f$ is proper, $f$ is onto. Let $n \geqq 2$, and let $w \in N^{n}$. Since $f$ is monotone onto, it is quasi-monotone [32, pp. 151-152], so that each component of $M^{n}-f^{-1}(w)$ has image $N^{n}-\{w\}$. Thus $f^{-1}(w)$ does not separate $M^{n}$. Hence $f$ satisfies conclusion (1).

Suppose $A_{1}=\varnothing$. If $M^{n} \neq B_{f}$, then there exists an open set $Q$ on which $f$ is a homeomorphism; since $\operatorname{dim}\left(f\left(R_{n-1}(f)\right)\right) \leqq n-1, f(Q)$ meets $N^{n}-f\left(R_{n-1}(f)\right)$, and a contradiction results. Thus conclusion (2)(i) holds in this case. In particular $f$ is not onto. For $y \in f\left(M^{n}\right)-f\left(R_{n-2}(f)\right)$ the Jacobian matrix of the restriction of $\rho f \sigma$ to a submanifold $\{x\} \times S(0, \varepsilon)$ of $S^{1} \times S(0, \varepsilon)$ has maximal rank. Also each restriction map $\rho f \sigma \mid\left(S^{1} \times\{t\}\right)$ is constant, and thus $\rho f \sigma\left(S^{1} \times S(0, \varepsilon)\right)$ is $C^{2}$ diffeomorphic to $S(0, \varepsilon)$, and $\rho f \sigma$ is the natural projection map. Conclusion (2)(ii) follows.

2.3. Remarks. Note that if $M^{n}$ and $N^{n}$ are oriented, then [28, p. 127, Theorem 4.2] a $C^{2}$ monotone map $f$ is onto if and only if the degree of $f$ is \pm 1 , and is not onto if and only if $\operatorname{deg} f=0$.

If $f$ is $C^{n}$ monotone and open, then $f$ is a homeomorphism. This statement is an immediate consequence of the structure theorem for proper open maps [7, p. 91].

2.4. Remark. Let $M^{n}$ and $N^{n}$ be $C^{\infty}$ manifolds, and let $f: M^{n} \rightarrow N^{n}$ be a proper map $C^{\infty}$ except on $f^{-1}\left(y_{i}\right)(i=1,2, \ldots, k)$. By the argument of [7, p. 95, (3.3)] there is a homeomorphism $h: N^{n} \rightarrow N^{n}$ such that $h f$ is $C^{\infty}$ and the restriction $h \mid\left[N^{n}-\bigcup_{i=1}^{k}\left\{y_{i}\right\}\right]$ is a $C^{\infty}$ diffeomorphism.

2.5. EXAMPLES. Differentiable monotone maps which are not acyclic (integral Cech cohomology). Let $p \in S^{k}, q \in S^{m}, r \in S^{k+m}$; and let $S^{k} \vee S^{m}$ be the subset $\left(\{p\} \times S^{m}\right) \cup\left(S^{k} \times\{q\}\right) \subset S^{k} \times S^{m}$. There is a $C^{\infty}$ diffeomorphism $g$ of $S^{k} \times S^{m}$ $-\left(S^{k} \vee S^{m}\right)$ onto $S^{k+m}-\{r\}$; define $f: S^{k} \times S^{m} \rightarrow S^{k+m}$ by $f\left(S^{k} \vee S^{m}\right)=\{r\}$ and elsewhere $f$ is $g$ (topologically, $f$ is the smash product map). Then $f$ is $C^{\infty}$ except on $f^{-1}(r)$, and by (2.4) it may be supposed to be $C^{\infty}$. In particular, we observe that the hypothesis $\operatorname{dim}\left(B_{f}\right) \leqq n-2$ is not sufficient to imply that $f$ is acyclic if $n \geqq 4$ (see (1.1)).

2.6. THEOREM. If $f$ is $C^{2}$ quasi-monotone, then there exists a natural number $k$ such that: (a) for every $y \in N^{n}, f^{-1}(y)$ has at most $k$ components; and (b) for every $y \in N^{n}-f\left(R_{n-2}(f)\right), f^{-1}(y)$ has exactly $k$ components, each a point or a $C^{2}$ embedding of $[0,1]$.

Suppose that $M^{n}$ and $N^{n}$ are oriented. (c) If the Jacobian determinant $J \geqq 0$ or $J \leqq 0$ at every point of $M^{n}$, then $k=|\operatorname{deg} f|$. (d) If $f$ is monotone, then $J \geqq 0$ or $J \leqq 0$ at every point.

Proof. If $f: M^{1} \rightarrow N^{1}$ is a proper quasi-monotone map, then either $M^{1}=N^{1}$ $=E^{1}$ or $M^{1}=N^{1}=S^{1}$. In the former case $f$ is monotone onto; in the latter case $f=h g$, where $g: S^{1} \rightarrow S^{1}$ is monotone onto, and $h: S^{1} \rightarrow S^{1}$ is a finite-to-one covering map [32, p. 153, (8.4)]. In either case the conclusions of the theorem are satisfied, so that we may suppose that $n \geqq 2$. 
See (1.6). Let $y(i) \in M^{n}-f\left(R_{n-2}(f)\right)$ be distinct points, and let $\rho$ and $\sigma$ be the maps given by (2.1)(b) and (c) with $\rho f \sigma: L^{1} \times E^{n-1} \rightarrow S^{1} \times E^{n-1}$. Since

$$
\rho f \sigma \mid\left(L^{1} \times\{0\}\right): L^{1} \times\{0\} \rightarrow S^{1} \times\{0\}
$$

is quasi-monotone [32, p. 152, (*)] and $\rho(y(i)) \in S^{1} \times\{0\}, \sigma^{-1}\left(f^{-1}(y(i))\right)$ have the same number of components $(i=1,2)$. Conclusion (b) follows.

Suppose that $u \in f\left(R_{n-2}(f)\right)$ and $f^{-1}(u)$ has (at least) $k+1$ components, $U_{i}$ $(i=1,2, \ldots, k+1)$. There exists an open $n$-cell $E$ about $u$ such that the $U_{i}$ are contained in different components of $f^{-1}(E)$; since $f$ is quasi-monotone, for each $y \in E, f^{-1}(y)$ has at least $k+1$ components. Since $\operatorname{dim}\left(f\left(R_{n-2}(f)\right)\right) \leqq n-2[25$, p. 173, Theorem 2], a contradiction of the choice of $k$ results. Thus (a) is proved.

Now suppose that $M^{n}$ and $N^{n}$ are connected and oriented. Since $\operatorname{dim}\left(f\left(R_{n-1}(f)\right)\right)$ $\leqq n-1$, there exists $y \in N^{n}-f\left(R_{n-1}(f)\right) ; f^{-1}(y)$ consists of exactly $k$ points, and if $J \geqq 0$ or $J \leqq 0$ at every point of $M^{n}$, then $k=|\operatorname{deg} f|$ [28, p. 127, Theorem 4.2].

Suppose that $M^{n}$ and $N^{n}$ are connected and oriented, and that $f$ is monotone. If $f$ is not onto, then $J \equiv 0((2.2),(2 \mathrm{i}))$; thus we may suppose that $f$ is onto, and thus quasi-monotone [32, p. 151]. Suppose that there exist points $x_{1}$ and $x_{2}$ in $M^{n}$ at which $J>0$ and $J<0$, respectively. Since $f$ is monotone, $f\left(x_{i}\right) \notin f\left(R_{n-1}(f)\right)$. Let $\rho$ and $\sigma$ be the maps given by (2.1)(b) and (c) for $y(i)=f\left(x_{i}\right)$. Since $f$ is monotone onto, $L^{1}=S^{1}$ and $\rho f \sigma \mid\left(S^{1} \times\{0\}\right)$ is monotone onto. Its derivative does not change sign, so the Jacobian determinant of $\rho f \sigma$ does not change sign on $S^{1} \times\{0\}$, and a contradiction of the choice of the $x_{i}$ results. Thus (d) is proved.

2.7. Remarks. Conclusion (d) cannot be extended to quasi-monotone maps $[6,(12)]$ (but see $[6,(3)$ and (4)]). The same example shows that conclusion (c) is false if $J$ changes sign.

In case $n=2(2.6)$ is related to [34, p. 665, (3.8) and (3.9)] and to [34, p. 671, (4.7)]. One can show by example that (2.6) is false for $C^{\infty}$ quasi-monotone proper maps $f: M^{m} \rightarrow N^{n}$ where $m>n$; in particular, the number of components of $f^{-1}(y)$ for $y$ a regular value (i.e., $y \in N^{n}-f\left(R_{n-1}\right)$ ) is not independent of $y$.

If $f$ is $C^{2}$ quasi-monotone, then it follows from (b) and [8, p. 371, (2.1)] that the restriction map $f \mid\left[M^{n}-f^{-1}\left(f\left(R_{n-2}(f)\right)\right)\right]$ has the factorization of [8].

2.8. Corollary. If $M^{n}$ and $N^{n}$ are oriented, $f$ is $C^{2}, \operatorname{dim}\left(B_{f}\right) \leqq n-2$, and $\operatorname{deg} f= \pm 1$, then $f$ is monotone (onto).

Proof. Since $\operatorname{dim}\left(B_{f}\right) \leqq n-2$, the Jacobian determinant $J \geqq 0$ or $J \leqq 0$; thus [28, p. 127, Theorem 4.2] for each $y \in N^{n}-f\left(R_{n-1}\right), f^{-1}(y)$ consists of exactly one point. Since $f$ is quasi-monotone $[8$, p. $380,(3.2)]$, in $(2.6) k=1$, so that $f$ is monotone onto.

2.9. REMARK. If $f$ is $C^{2}$ with $\operatorname{dim}\left(B_{f}\right) \leqq n-2$ and $N^{n}$ is orientable, then $M^{n}$ is orientable.

Without the hypothesis that $\operatorname{dim}\left(B_{f}\right) \leqq n-2$, the last statement is false (2.15). The covering of the projective plane is a counterexample to the converse statement. 
Proof. See (1.5). Suppose $M^{n}$ is not orientable; then there is a simplicial map $\alpha: S^{1} \rightarrow M^{n}$ around which the orientation changes, and we may suppose that $\alpha\left(S^{1}\right) \cap B_{h}=\varnothing$. The orientation of $N^{n}$ changes around $f \alpha$, and a contradiction results.

2.10. The proof of (1.3). See (1.6). We may suppose that $\operatorname{deg} f>0$. We first prove the theorem in case $n=1$. Let $y \in N^{1}$, and let $J \subset N^{1}$ be a closed interval such that $y \in$ int $J$ and the endpoints $a_{r}$ of $J(r=1,2)$ are regular values [28, p. 47, Theorem 3.1]. Let $A_{s}(s=1,2, \ldots, m)$ be the components of $f^{-1}$ (int $\left.J\right)$; since $\operatorname{deg} f>0$, each $\bar{A}_{s}$ is a closed interval. From [28, p. 127, Theorem 4.2] $\operatorname{deg} f=\sum_{s}$ $\operatorname{deg}\left(f \mid A_{s}\right)$, and to prove that $f^{-1}(y)$ has at least $\operatorname{deg} f$ components, it suffices to prove the corresponding result for each map $f \mid A_{s}: A_{s} \rightarrow$ int $J$. Since $f$ is proper, $f^{-1}\left(\left\{a_{1}, a_{2}\right\}\right) \cap \bar{A}_{s}$ consists of the two endpoints of $\bar{A}_{s}$. If, for either $r, f^{-1}\left(a_{r}\right)$ consists of both endpoints, then $\operatorname{deg}\left(f \mid A_{s}\right)=0$, and the conclusion is vacuously satisfied; otherwise, $\operatorname{deg}\left(f \mid A_{s}\right)= \pm 1$ and $f\left(A_{s}\right)=J$, so that again the conclusion is clearly satisfied.

Now suppose that $n \geqq 2$, and there is a point $y \in N^{n}-f\left(R_{n-2}(f)\right)$ such that $f^{-1}(y)$ has less than $\operatorname{deg} f$ components. Let $y_{1}$ be a regular value of $f ; f^{-1}\left(y_{1}\right)$ consists of a finite number $j$ of points, and by [28, p. 127, Theorem 4.2] $j \geqq \operatorname{deg} f$. In particular, $y_{1} \neq y$. Let $\sigma$ and $\rho$ be the maps given by $(2.1)(\mathrm{c})$ for $y$ and $y_{1}$; then $\rho f \sigma: L^{1} \times E^{n-1} \rightarrow S^{1} \times E^{n-1}$ with $\rho f \sigma\left(L^{1} \times\{t\}\right) \subset S^{1} \times\{t\}$ for each $t \in E^{n-1}$. Let $L_{s}(s=1,2, \ldots, m)$ be the components of $L^{1}$. Since $S^{1} \times\{0\}$ contains a regular value of $\rho f \sigma$ (namely, $\rho\left(y_{1}\right)$ ), orientations can be defined on $S^{1}$ and $L_{s}$ so that $\sum_{s} \operatorname{deg}\left(\rho f \sigma \mid\left(L_{s} \times\{0\}\right)\right)=\operatorname{deg} f$. Since the theorem is true for $n=1, \sigma^{-1}\left(f^{-1}(y)\right)$ (and therefore $f^{-1}(y)$ ) has at least $\operatorname{deg} f$ components.

Analogous questions for simplicial maps are discussed by Hopf in [14].

2.11. THEOREM. Let $M^{n}$ be compact, let $n \geqq 2$, let $f$ be $C^{2}$ quasi-monotone, and let $V$ be an m-dimensional vector space over a field $F$. Let $k$ be the natural number of (2.6), and let $r=\operatorname{dim}\left(H^{n-1}\left(M^{n} ; V\right)\right)$.

(a) Then $H^{n-1}\left(f^{-1}(y) ; V\right)=0$ for all but at most $m k+r$ points $y \in N^{n}$.

(b) If $M^{n}$ is orientable or if $F=Z_{2}$, then $H^{n-1}\left(f^{-1}(y) ; V\right)=0$ for all but at most $m(k-1)+r$ points $y \in N^{n}$; in particular, if $f$ is monotone onto, then

$$
i^{*}: H^{n-1}\left(M^{n} ; V\right) \rightarrow \sum_{y \in N^{n}} H^{n-1}\left(f^{-1}(y) ; V\right),
$$

(induced by inclusion) is an epimorphism.

Proof. Suppose that there are distinct points $y_{s}(s=1,2, \ldots, t)$ such that $H^{n-1}\left(f^{-1}\left(y_{s}\right) ; V\right) \neq 0$; let $Y=\bigcup_{s} f^{-1}\left(y_{s}\right)$. Since $H^{n-1}(Y ; V) \approx \sum_{s} H^{n-1}\left(f^{-1}\left(y_{s}\right) ; V\right)$, $\operatorname{dim}\left(H^{n-1}(Y ; V)\right) \geqq t$; from the exactness of the cohomology sequence of $\left(M^{n}, Y\right)$, $\operatorname{dim}\left(H^{n-1}(Y ; V) /\right.$ imag $\left.i^{*}\right) \geqq t-r$, so that $\operatorname{dim}\left(\operatorname{ker} j^{*}\right) \geqq t-r$. Since $f$ is quasimonotone, $M^{n}-Y$ has at most $k$ components $(2.6)$; thus $\operatorname{dim}\left(H^{n}\left(M^{n}, Y ; V\right)\right) \leqq m k$. Since $\operatorname{ker} j^{*}$ is a subspace, $m k \geqq t-r$, i.e., $m k+r \geqq t$. 
If $M^{n}$ is orientable, or if $F=Z_{2}$, then $H^{n}\left(M^{n} ; V\right) \approx V, H^{n}\left(M^{n}, Y ; V\right)$ is isomorphic to the direct sum of at most $k$ copies of $V$, and $\operatorname{ker} j^{*}$ is isomorphic to the direct sum of at most $k-1$ copies of $V$; thus $m(k-1)+r \geqq t$. If in addition $f$ is monotone, then $j^{*}$ is an isomorphism, so that $i^{*}$ is an epimorphism.

The theorem is a generalization of [8, p. 372, (2.3)]. Example (2.14) with $V=Z_{3}$ shows that the orientability hypothesis is required in (b).

2.12. Example. No analogous statement can be made for $H^{k}\left(M^{n}\right)$ with $k=1,2$, $\ldots, n-2$. Let $g: S^{1} \times S^{1} \rightarrow S^{2}$ be the map given in (2.5) (for $k=m=1$ ), and let $\rho_{i}: S^{1} \times S^{1} \rightarrow S^{1}$ be the projection maps $(i=1,2)$. Let $M_{i}$ be the mapping cylinder of $\rho_{i}$ (a solid torus), let $N_{i}$ be the mapping cylinder of a constant map on $S^{2}$ (i.e., a cone over $S^{2}$ ), and let $M^{3}$ (resp., $N^{3}$ ) be the natural union of the $M_{i}$ (resp., $N_{i}$ ), $i=1,2$; then $M^{3}$ and $N^{3}$ are each diffeomorphic to $S^{3}$. Let $f: M^{3} \rightarrow N^{3}$ be the map induced by $g$. Since $g$ is $C^{\infty}, f$ is $C^{\infty}$ except at $f^{-1}\left(q_{i}\right)$, where $q_{i}(i=1,2)$ are the poles of $N^{3}$ as a suspension over $S^{2}$. From (2.4) we may suppose that $f$ is $C^{\infty}$. For uncountably many points $y \in N^{3}, f^{-1}(y)$ is homeomorphic to $S^{1} \vee S^{1}$; thus $H^{1}\left(f^{-1}(y) ; Z_{2}\right) \approx Z_{2} \oplus Z_{2}$ while $H^{1}\left(M^{3} ; Z_{2}\right)=0$.

Analogous examples are obtained from the other maps of (2.5), and suspensions of them.

2.13. REMARK. Let $M^{n}$ be oriented, let $f$ be $C^{1}$ monotone onto, let $G$ be a principal ideal domain, and let Čech homology and cohomology with compact supports be denoted by $H_{k}^{c}$ and $H_{c}^{k}$, respectively. Then

$$
0 \longrightarrow \operatorname{ker} f_{*} \longrightarrow H_{k}^{c}\left(M^{n} ; G\right) \stackrel{f_{*}}{\longrightarrow} H_{k}^{c}\left(N^{n} ; G\right) \longrightarrow 0
$$

and

$$
0 \longrightarrow H_{c}^{k}\left(N^{n} ; G\right) \stackrel{f .}{\longrightarrow} H_{c}^{k}\left(M^{n} ; G\right) \longrightarrow \operatorname{coker} f^{*} \longrightarrow 0
$$

are split exact sequences $(k=0,1, \ldots)$.

By Sard's Theorem [28, p. 47, Theorem 3.1] the hypothesis of [18, p. 639, Theorem 3] is satisfied, and the remark is an immediate consequence of (the proof of) conclusion (1) of that theorem ((1) follows from the naturality of the Poincare Duality (cap product) isomorphism).

2.14. ExAmple. The hypothesis that $M^{n}$ is oriented is necessary. Let $S^{1}$ be the canonical circle in the projective plane $P^{2}$, and let $f: P^{2} \rightarrow S^{2}$ be the monotone (onto) map for which $B_{f}=S^{1}$ and $f\left(S^{1}\right)$ is a point. From (2.4) we may suppose that $f$ is $C^{\infty}$, while (a) is not satisfied for $k=2$. See also (2.9).

2.15. EXAMPLE. In view of (2.11) it is natural to ask whether in $(2.13$ (b)) for $k=(n-1)$ coker $f^{*}$ is $\sum_{y \in N^{n}} H^{n-1}\left(f^{-1}(y) ; G\right)$, i.e., is the sequence

$$
0 \longrightarrow H^{n-1}\left(N^{n} ; G\right) \stackrel{f^{*}}{\longrightarrow} H^{n-1}\left(M^{n} ; G\right) \stackrel{i^{\bullet}}{\longrightarrow} \sum_{y \in N^{n}} H^{n-1}\left(f^{-1}(y) ; G\right) \longrightarrow 0
$$

exact? The following example provides a negative answer. 
Let $T^{2}$ be the torus, let $g: T^{2} \rightarrow S^{2}$ be the map given in (2.5) for $k=m=1$, and let $f: T^{2} \times S^{1} \rightarrow S^{2} \times S^{1}$ be defined by $f(u, v)=(g(u), v)$. Then

$$
\sum_{y \in S^{2} \times S^{1}} H^{2}\left(f^{-1}(y) ; Z_{2}\right)=0
$$

while $H^{2}\left(T^{2} \times S^{1} ; Z_{2}\right)$ is not isomorphic to $H^{2}\left(S^{2} \times S^{1} ; Z_{2}\right)$.

3. Technical lemmas. The results of this section are needed for $\S 4$.

Given $C^{r}$ manifolds $M^{m}$ and $N^{n}$, let $\mathbf{C}^{r}\left(M^{m}, N^{n}\right)$ be the space of $C^{r}$ maps $f: M^{m} \rightarrow N^{n}$ with the fine $C^{r}$ topology $(r=0,1, \ldots)$. (The fine and coarse $C^{r}$ topologies are defined in [22, pp. 25-28].) Let $\mathfrak{R}^{r}\left(M^{m}\right)$ be the set of those open neighborhoods $\boldsymbol{U}$ of the identity map $I \in \mathbf{C}^{r}\left(M^{m}, M^{m}\right)$ such that, if $\chi \in \boldsymbol{U}$, then $\chi$ is a diffeomorphism [22, p. 29, (3.10)].

3.1. Lemma. Given the hypotheses of [22, p. 40, (4.7)], let $U \in \mathfrak{N}^{r}\left(E^{n}\right)$, and let $Z \subset E^{n}$ be a bounded open neighborhood of $f(\bar{V})$. Then there is a $C^{r}$ diffeomorphism $\chi \in \boldsymbol{U}$ such that

(a) $\chi f=h$ satisfies the conclusions of $[22$, p. $40,(4.7)]$,

(b) $\chi$ is the identity map I off $Z$, and

(c) $\chi\left(\{t\} \times E^{n-m}\right)=\{t\} \times E^{n-m}$ for each $t \in E^{m}$.

Proof. In $[22$, p. $35,(4.1)] f_{1}$ may be chosen to approximate $f$ in the coarse $C^{r}$ topology.

With $g, \pi$, and $\mathcal{O}$ as in the proof of $[22$, p. $40,(4.7)]$ and $\alpha>0$, define

$$
A_{\alpha}=\left\{x \in \pi^{-1}(\mathcal{O}):|x-g(\pi(x))|<\alpha\right\}
$$

and choose $\alpha>0$ such that the closure $\operatorname{Cl}\left[A_{\alpha} \cap \pi^{-1}(\pi(f(V)))\right] \subset Z$. Let $X=A_{\alpha}$, and let $Y=E^{n}-\mathrm{Cl}\left[A_{\beta} \cap \pi^{-1}(\pi(f(V)))\right]$ for any $\beta$ with $0<\beta<\alpha$.

Let $\{\phi, 1-\phi\}$ be a $C^{\infty}$ partition of unity dominated by the open cover $\{X, Y\}$ of $E^{n}$. We may suppose that $\boldsymbol{U}$ is sufficiently small that if $\chi \in U$, then $\chi(f(\bar{V})) \subset A_{\beta}$. For any $C^{r} \operatorname{map} \psi: E^{n} \rightarrow E^{n}$ the map $\chi_{\psi}$ defined by $\chi_{\psi}(x)=\phi(x) \cdot \psi(x)+(1-\phi(x)) \cdot x$ agrees with $I$ off the compact set $\bar{X}$; thus there is a neighborhood $V$ of $I$ in the coarse $C^{r}$ topology such that, if $\psi \in V$, then $\chi_{\psi} \in \boldsymbol{U}$.

In the proof of [22, (4.7)] define $\psi: E^{n} \rightarrow E^{n}$ by $\psi(x)=x+\tilde{g}(\pi(x))-g(\pi(x))$ for $x \in \pi^{-1}(\mathcal{O})$, and $\psi(x)=x$ elsewhere. If $\delta$ is chosen sufficiently small, and $\tilde{g}_{0}$ is chosen to be an $\varepsilon$-approximation to $g_{0}$ in the coarse $C^{r}$ topology, then $\psi$ will be in $V$; moreover $\psi\left(\{t\} \times E^{n-m}\right)=\{t\} \times E^{n-m}$ for each $t \in E^{m}$. The map $\chi=\chi_{\downarrow}$ is thus a $C^{r}$ diffeomorphism satisfying conclusion (c).

By its definition $\chi$ is the identity off $X$; since $\psi$ is the identity off $\pi^{-1}(\pi(f(V)))$, it follows that $\chi$ is also. Thus $\chi$ satisfies conclusion (b) also. Since $\chi(f(\bar{V})) \subset A_{\beta}$ and $\chi$ satisfies conclusion (c), $\chi(f(\bar{V})) \cap \bar{Y}=\varnothing$; since $\psi=I$ off $\pi^{-1}(\pi(f(V))), \chi f=\dot{\psi} f$, and one may readily verify that $\chi f$ satisfies the conclusions of $[22,(4.7)]$. 
3.2. Lemma. Let $L^{p}$ and $M^{n-p}$ be $C^{\infty}$ manifolds, let $V \in \mathfrak{R}^{r}\left(L^{p} \times M^{n-p}\right)$, and let $\Gamma^{q}(q \geqq n-p)$ be a $C^{r}(r=1,2, \ldots)$ submanifold of $L^{p} \times M^{n-p}$ transverse to $L^{p} \times\{t\}$ for each $t \in M^{n-p}$. Then there exists $\psi \in V$ such that:

(a) $\psi\left(\Gamma^{q}\right)$ is a $C^{\infty}$ submanifold of $L^{p} \times M^{n-p}$;

(b) $\psi\left(\Gamma^{q}\right)$ is transverse to $L^{p} \times\{t\}$ for each $t \in M^{n-p}$;

(c) $\psi\left(L^{p} \times\{t\}\right)=L^{p} \times\{t\}$ for each $t \in M^{n-p}$.

The manifold $M^{n-p}$ may be a single point, in which case the transversality condition is vacuous; thus the lemma includes [8, p. 376, (2.5)].

Proof. Given $(x, t) \in \Gamma^{q}, x \in L^{p}$ and $t \in M^{n-p}$, let $(P \times Q, \alpha \times \beta)$ be a $C^{\infty}$ coordinate pair, where $P$ (resp., $Q$ ) is an open neighborhood of $x$ (resp., $t$ ) in $L^{p}$ (resp., $M^{n-p}$ ) and $\alpha$ (resp., $\beta$ ) is a $C^{\infty}$ diffeomorphism of $P$ (resp., $Q$ ) onto $\alpha(P) \subset E^{p}$ (resp., $\beta(Q) \subset E^{n-p}$ ) with $\alpha(x)=0$ (resp., $\beta(t)=0$ ). Since $\Gamma^{q}$ is transverse to $L^{p} \times\{t\}$ at $(x, t)$, there is a coordinate plane $E^{p+q-n} \subset E^{p}$ (if $q=n-p$, then $E^{p+q-n}$ is the origin $O$ of $\left.E^{p}\right)$ and a neighborhood $T \subset \Gamma^{q}$ of $(x, t)$ such that $T \subset P \times Q$ and the projection of $\alpha \times \beta(T)$ onto $E^{p+q-n} \times E^{n-p}$ is a $C^{r}$ embedding. Let $\pi: E^{n} \rightarrow E^{q}=E^{p+q-n} \times E^{n-p}$ be projection. Let $(R, \gamma)$ be a $C^{r}$ coordinate pair of $\Gamma^{q}$, i.e., $\gamma$ is a $C^{r}$ diffeomorphism of $R$ onto $\gamma(R) \subset E^{q}$, with $(x, t) \in R, \bar{R}$ compact, and $\bar{R} \subset T$.

The sets $R$ for $(x, t) \in \Gamma^{q}$ cover $\Gamma^{q}$, and so there is a locally finite subcover $R_{i}$ $(i=1,2, \ldots)$; let $P_{i}, Q_{:}, T_{i}, \alpha_{i}, \beta_{i}, \gamma_{i}, \pi_{i}$ be the sets and functions thus defined. Let $U_{i}=\gamma_{i}\left(R_{i}\right) \subset E^{q}$, and let $V_{i}$ and $W_{i}$ be open subsets of $U_{i}$ such that $\bar{W}_{i} \subset V_{i}, \bar{V}_{i} \subset U_{i}$, and the sets $\gamma_{i}^{-1}\left(W_{i}\right)$ cover $\Gamma^{q}\left[22\right.$, p. 7]. Let $X_{i}$ be an open subset of $L^{p} \times M^{n-p}$ such that $\bar{X}_{i} \subset P_{i} \times Q_{i}, \gamma_{i}^{-1}\left(\bar{V}_{i}\right) \subset X_{i}, \bar{X}_{i} \cap \Gamma^{q} \subset \gamma_{i}^{-1}\left(U_{i}\right)$, and the sets $\bar{X}_{i}$ are locally finite $(i=1,2, \ldots)$.

We may suppose that $\boldsymbol{V}$ is sufficiently small that, for each $\psi \in V$, conclusion (b) is satisfied, $\psi\left(\bar{X}_{i}\right) \subset P_{i} \times Q_{i}$, and $\pi_{i} \circ\left(\alpha_{i} \times \beta_{i}\right) \circ \psi \circ \gamma_{i}^{-1}$ is an embedding. Moreover we may suppose that $V$ is a basis neighborhood $\left(X\left(f, \delta_{i}\right)\right.$ in $[22$, p. 26]).

We will define $\psi_{i} \in V\left(i=0,1, \ldots ; \psi_{0}=I\right)$ such that (1) $\psi_{i}$ agrees with $\psi_{i-1}$ off $\bar{X}_{i}$, (2) $\psi_{i}\left(\bigcup_{j \leqq i} \gamma_{j}^{-1}\left(W_{j}\right)\right)$ is a $C^{\infty}$ submanifold of $L^{p} \times M^{n-p}$, and (3) $\psi_{i}\left(L^{p} \times\{t\}\right)$ $=L^{p} \times\{t\}$ for each $t \in M^{n-p}$. Because the $\bar{X}_{i}$ are locally finite, the limit map $\psi$ is a well-defined $C^{r}$ map; since each $\psi_{i}$ is in the basis neighborhood $\boldsymbol{V}, \psi \in V$ also. The remaining properties of $\psi$ follow immediately. (Note that $\psi_{\imath}+\psi$ in the fine $C^{r}$ topology necessarily!)

The construction of the maps $\psi_{i}$ is by induction; $\psi_{0}=I$; suppose that $\psi_{i-1}$ has been defined. Let $f=\left(\alpha_{i} \times \beta_{i}\right) \circ \psi_{i-1} \circ \gamma_{i}^{-1}$, let $Z \subset E^{n}$ be $\alpha_{i} \times \beta_{i}\left(\psi_{i-1}\left(X_{i}\right)\right)$, and let $\chi \in \boldsymbol{U}$ be given by (3.1), where $\boldsymbol{U} \in \mathscr{N}^{r}\left(E^{n}\right)$ is to be defined. For $x \notin X_{i}$ let $\psi_{i}(x)$ $=\psi_{i-1}(x)$; for $x \in \bar{X}_{i}$, let

$$
\psi_{i}(x)=\left(\alpha_{i} \times \beta_{i}\right)^{-1} \circ \chi \circ\left(\alpha_{i} \times \beta_{i}\right) \circ \psi_{i-1}(x) .
$$

Then $\psi_{i}$ is a well-defined $C^{r}$ map, and if the $\boldsymbol{U}$ of (3.1) is sufficiently small, $\psi_{i} \in \boldsymbol{V}$. Properties (1) and (3) follow readily. Since $\bar{X}_{i} \cap \Gamma^{q} \subset \gamma_{i}^{-1}\left(U_{i}\right)$, it follows from (1) 
and the inductive hypothesis that

$$
\psi_{i}\left(\left(\bigcup_{j \leqq i} \gamma_{j}^{-1}\left(W_{j}\right)\right)-\gamma_{i}^{-1}\left(U_{i}\right)\right)
$$

is a $C^{\infty}$ submanifold of $L^{p} \times M^{n-p}$; that

$$
\psi_{i}\left(\gamma_{i}^{-1}\left(U_{i}\right) \cap \bigcup_{j \leqq i} \gamma_{j}^{-1}\left(W_{j}\right)\right)
$$

is $C^{\infty}$ also follows from (3.1)(a)(3) and (a)(4).

3.3. Lemma. Let $L^{p}$ be a compact $C^{r}$ manifold $(p=1,2, \ldots, n-1 ; r=1,2, \ldots$ or $\left.r=\infty ; \partial L^{p}=\varnothing\right)$, and let $A_{i} \subset L^{p} \times E^{n-p}(i=1,2, \ldots, m)$ be $C^{r}$ submanifolds with $\partial A_{i}=\varnothing$ such that:

(a) each $A_{i}$ is a closed subset of $L^{p} \times E^{n-p}$,

(b) each $A_{i}$ is transverse to $L^{p} \times\{t\}$ (for each $t \in E^{n-p}$ ),

(c) each $A_{i} \cap\left(L^{p} \times\{0\}\right)=K_{i}$ is a compact $C^{r}$ manifold, $\partial K_{i}=\varnothing$, and

(d) the $A_{i}$ are mutually disjoint.

Then there is a $C^{r}$ diffeomorphism $\eta$ of $L^{p} \times E^{n-p}$ onto itself such that $\eta\left(K_{i} \times E^{r}\right)=A_{i}$ and $\eta\left(L^{p} \times\{t\}\right)=L^{p} \times\{t\}\left(t \in E^{n-p} ; i=1,2, \ldots, m\right)$.

The manifolds $A_{i}$ may have different dimensions; a useful case is that for which $A_{i}=\partial B_{i}$ and $\operatorname{dim}\left(B_{i}\right)=n$.

Proof. See (1.5). We may suppose that $L^{p}$ is a $C^{\infty}$ Riemannian manifold and that $L^{p} \times E^{n-p}$ has the product Riemannian metric. By (3.2) we may suppose that $r=\infty$; let $q_{i}=\operatorname{dim}\left(A_{i}\right)$.

For each $(x, t) \in A_{k}$ there is $U(x, t)$ open in $L^{p} \times E^{n-p}$ such that $(x, t) \in U(x, t)$, $U(x, t) \cap A_{k}$ is a $C^{\infty}$ diffeomorph of $\operatorname{int}\left(D^{q_{k}}\right)$, and $U(x, t) \cap A_{i}=\varnothing$ for $i \neq k$. The sets $U(x, t)$ cover $\bigcup_{i} A_{i}$; let $U_{r}(r=1,2, \ldots)$ be a locally finite subcover, where $U_{r}$ meets (only) $A_{k(r)}$. Since the normal bundle of $U_{r} \cap A_{k(r)}$ in $U_{r}$ is trivial, there is a tubular neighborhood $V_{r}$ of $U_{r} \cap A_{k(r)}$ and a $C^{\infty}$ diffeomorphism $\rho_{r}$ of $V_{r}$ onto $\operatorname{int}\left(D^{\left.q_{k(r)}\right)} \times E^{n-q_{k(r)}}\right.$; let $\pi_{r}$ be the projection of $D^{q_{k(r)}} \times E^{n-q_{k(r)}}$ onto $E^{n-q_{k(r)}}$. For each $s \in E^{n-q_{k(r)}}, \rho^{-1}\left(\pi^{-1}(s)\right)$ is an open $q_{k(r)}$-cell, and by choosing $V_{r}$ sufficiently small about $U_{r} \cap A_{k(r)}$, we may suppose that $\rho^{-1}\left(\pi^{-1}(s)\right)$ is transverse to $L^{p} \times\{t\}$ for each $t \in E^{n-p}$. For each $(x, t) \in V_{r}$, let $J_{r}(x, t)$ be that set $\rho^{-1}\left(\pi^{-1}(s)\right)$ containing $(x, t)$.

Let $t^{j}(j=1,2, \ldots, n-p)$ be the usual coordinates on $E^{n-p}$, and let $\partial / \partial t^{j}$ be the corresponding vector fields in $L^{p} \times E^{n-p}$. For $(x, t) \in V_{r}$ let $P_{r}(x, t)$ be the $((n-p)$-dimensional) vector space orthogonal to the tangent space

$$
T_{(x, t)}\left(J_{r}(x, t) \cap\left(L^{p} \times\{t\}\right)\right)
$$

in $T_{(x, t)} J_{r}(x, t)$. Since

$$
T_{(x, t)}\left(L^{p} \times E^{n-p}\right)=T_{(x, t)} J_{r}(x, t)+T_{(x, t)}\left(L^{p} \times\{t\}\right),
$$

it is the direct sum $P_{r}(x, t) \oplus T_{(x, t)}\left(L^{p} \times\{t\}\right)$. It follows that orthogonal projection 
of $P_{r}(x, t)$ onto $T_{(x, t)}\left(\{x\} \times E^{n-p}\right)$ is an isomorphism; let $u_{j, r}(x, t)$ be the vector in $P_{r}(x, t)$ which projects onto $\partial / \partial t^{j}(x, t)$. Then $u_{j, r}$ is a $C^{\infty}$ vector field with domain $V_{\mathrm{r}}(j=1,2, \ldots, n-p ; r=1,2, \ldots)$.

Let $V_{0}=\left(L^{p} \times E^{n-p}\right)-\bigcup_{i} A_{i}$, let $\beta_{r}(r=0,1, \ldots)$ be a $C^{\infty}$ partition of unity dominated by $V_{r}$, and let

$$
v_{j}=\beta_{0} \partial / \partial t^{j}+\sum_{r=1}^{\infty} \beta_{r} u_{j, r} \quad(j=1,2, \ldots, n-p) .
$$

Then the projection of each $v_{j}(x, t)$ on $T_{(x, t)}\left(\{x\} \times E^{n-p}\right)$ is $\partial / \partial t^{j}(x, t)$, and $v_{j}$ agrees with $u_{j, r}$ on $V_{r} \cap A_{k(r)}$. Let $\phi_{j, s}\left(j=1,2, \ldots, n-p ; s \in L^{p} \times E^{n-p}\right)$ be the one-parameter group of diffeomorphisms associated with $v_{j}[21, \mathrm{p} .10,(2.4)]$, and define $\eta$ by

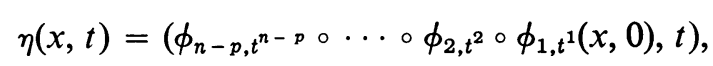

where $t=\left(t^{1}, t^{2}, \ldots, t^{n-p}\right)$ and composition is denoted by $\circ$.

3.4. Lemma. Let $f: M^{m} \rightarrow N^{n}$ be a $C^{k}$ proper map $(k=1,2, \ldots ; m \geqq n)$. Let $K_{i}(i=0,1, \ldots, s)$ be compact $C^{k}$ submanifolds of $N^{n}$ such that $\partial K_{i}=\varnothing, f$ is transverse regular on $K_{i}, K_{i} \subset K_{0}=K_{0}^{p}$, and the $K_{i}$ with $i>0$ are mutually disjoint; let $\rho$ be a $C^{k}$ diffeomorphism mapping a neighborhood of $K_{0}^{p}$ onto $K_{0}^{p} \times E^{n-p}$ with $\rho(x)=(x, 0)$ for $x \in K_{0}^{p}$.

Then there exist $\varepsilon>0$ and a $C^{k}$ diffeomorphism $\omega$ of $f^{-1}\left(K_{0}^{p}\right) \times S(0, \varepsilon)$ onto a neighborhood of $f^{-1}\left(K_{0}^{p}\right)$ such that for $h=\rho f \omega$ and each $t \in S(0, \varepsilon)$ and $i=0,1, \ldots, s$ :

(a) $h\left(f^{-1}\left(K_{0}^{p}\right) \times\{t\}\right)=K_{0}^{p} \times\{t\}$,

(b) $h^{-1}\left(K_{i} \times S(0, \varepsilon)\right)=f^{-1}\left(K_{i}\right) \times S(0, \varepsilon)$, and

(c) $h$ is transverse regular on $\partial K_{i} \times\{t\}$.

(d) If $K_{i}=\partial \Gamma_{i}^{p}$ with $\Gamma_{i}^{p} \subset K_{0}^{p}$, then

$$
h^{-1}\left(\Gamma_{i}^{p} \times S(0, \varepsilon)\right)=f^{-1}\left(\Gamma_{i}^{p}\right) \times S(0, \varepsilon) .
$$

By [29, p. 23] $f^{-1}\left(K_{i}\right)$ is a $C^{k}$ manifold; the dimensions of the $K_{i}$ may be different.

Proof. The proof of $[8$, p. $376,(2.7)]$ actually yields the stronger analogous result for maps $f: M^{q} \rightarrow N^{n}$ with $q \geqq n$; the dimension of $L$ is then $p+q-n$. Thus there are $\varepsilon>0$ and a $C^{k}$ diffeomorphism $\sigma$ of $f^{-1}\left(K_{0}^{p}\right) \times S(0, \varepsilon)$ onto a neighborhood of $f^{-1}\left(K_{0}^{p}\right)$ such that $\rho f \sigma\left(f^{-1}\left(K_{0}^{p}\right) \times\{t\}\right)=K_{0}^{p} \times\{t\}$ for each $t \in S(0, \varepsilon)$. For $\varepsilon$ sufficiently small $\rho f \sigma$ is transverse regular on $K_{i}^{p} \times\{t\}(t \in S(0, \varepsilon) ; i=1,2, \ldots, s)$, and thus is transverse regular on $K_{i}^{p} \times S(0, \varepsilon)$. Hence, for each $i$, either (1) $\sigma^{-1}\left(f^{-1}\left(\rho^{-1}\left(K_{i} \times S(0, \varepsilon)\right)\right)\right)$ is a $C^{k}$ manifold $A_{i}$ which is a closed subset of

$$
\sigma^{-1}\left(f^{-1}\left(\rho^{-1}\left(K_{0}^{p} \times S(0, \varepsilon)\right)\right)\right)=f^{-1}\left(K_{0}\right) \times S(0, \varepsilon)
$$

and is transverse to $f^{-1}\left(K_{0}^{p}\right)=\{t\}$ for each $t \in S(0, \varepsilon)$, or (2) it is empty.

There is a $C^{k}$ diffeomorphism $\eta$ of $f^{-1}\left(K_{0}^{p}\right) \times S(0, \varepsilon)$ onto itself given by (3.3) for all the $i$ satisfying (1). Let $\omega=\sigma \eta$; it follows readily that $\omega$ has the desired properties. 
3.5. LemMA. Let $f: M^{m} \rightarrow N^{n}$ be $C^{k}(k \geqq p+1)$, and let $\lambda: D^{p} \rightarrow N^{n}$ be a $C^{k}$ embedding with $f$ transverse regular on $\lambda\left(D^{p}\right)(p<n)$. Then there is a $C^{k}$ diffeomorph $\Sigma$ of $S^{p}$ such that $\lambda\left(D^{p}\right) \subset \Sigma$, the normal bundle of $\Sigma$ is trivial, and $f$ is transverse regular on $\Sigma$.

Proof. See (1.5). There is a $C^{k}$ embedding $\mu$ of $D^{n}$ (and, in fact, of $S(0,1+\varepsilon) \subset E^{n}$ for some $\varepsilon>0$ ) which extends $\lambda$ [24]. Thus there is a $C^{k}$ embedding $\nu: S^{p} \rightarrow N^{n}$ such that $\lambda\left(D^{p}\right) \subset \nu\left(S^{p}\right)$ and the normal bundle of $\nu\left(S^{p}\right)$ is trivial.

Let $T$ be a tubular neighborhood of $\nu\left(S^{p}\right)-\lambda\left(D^{p}\right)$ with $T \cap \lambda\left(D^{p}\right)=\varnothing$; by the proof of $\left[29\right.$, p. 26] there is a $C^{k}$ diffeomorphism $A$ of $N^{n}$ onto itself such that $A$ is the identity map off $T$, and $f$ is transverse regular on $A^{-1}\left(\nu\left(S^{p}\right)-\lambda\left(D^{p}\right)\right)$. Then $\Sigma=A^{-1}\left(\nu\left(S^{p}\right)\right)$ has the desired properties.

3.6. REMARK. The proof of [8, p. 382, (3.5)] actually shows the following: given $f: M^{n} \rightarrow N^{n}$ a $C^{m}$ proper map $(m, n \geqq 2)$ with $\operatorname{dim}\left(B_{f}\right) \leqq n-2$, there exists a $C^{m}$ proper map $h: M^{n} \rightarrow N^{n}$ such that (a) $B_{h} \subset B_{f}$, (b) $h\left(B_{h}\right) \subset h\left(R_{n-2}(h)\right.$ ), and (c) for each $y \in N^{n}, f^{-1}(y)$ and $h^{-1}(y)$ have the same number of components. Moreover, given any positive real-valued map $\delta$ defined on $M^{n}, h$ may be chosen so that, for each $x \in M^{n}, d(h(x), f(x))<\delta(x)$.

4. Differentiable acyclic maps. This section deals with sufficient conditions for a monotone map to be acyclic or cellular.

4.1. THEOREM. If $f$ is $C^{3}$ monotone with $\operatorname{dim}\left(B_{f}\right) \leqq n-2$, then the homomorphism $f_{*}: \pi_{1}\left(M^{n}\right) \rightarrow \pi_{1}\left(N^{n}\right)$ is an isomorphism (onto).

The condition on $B_{f}$ is necessary for $f_{*}$ to be a monomorphism ((2.14) and (4.5)).

Proof. If $n=1$ or 2 , then $f$ is a homeomorphism; thus we may suppose that $n \geqq 3$. See (1.5). The map $f$ is onto by (2.2).

Since $\operatorname{dim}\left(f\left(R_{n-1}(f)\right)\right) \leqq n-1[25$, p. 173, Theorem 2], we may choose the base points $x$ and $y$ for the fundamental groups so that $f(x)=y$ and $y \notin f\left(R_{n-1}(f)\right)$. The group $\pi_{1}\left(N^{n}, y\right)$ is generated by the polyhedral circles through $y$, and thus by the $C^{\infty}$ embeddings $\gamma: S^{1} \rightarrow N^{n}$ with $y \in \gamma\left(S^{1}\right)$. We may suppose that $f$ is transverse regular on $\gamma\left(S^{1}\right)\left[29\right.$, p. 26]; as a result $f^{-1}\left(\gamma\left(S^{1}\right)\right)$ is $C^{2}$ diffeomorphic to $S^{1}$ [29, p. 23], and defines an element of $\pi_{1}\left(M^{n}, x\right)$. Thus $f_{*}$ is an epimorphism (independent of hypothesis on $B_{f}$ ).

Now we prove that $f_{*}$ is a monomorphism. For each $x \in M^{n}$ let $\delta(x)=r(f(x))$, where $r$ is a positive continuous function on $N^{n}$ less than the number of [17, p. 165, (3.4)]; let $h$ be the $C^{3}$ map of (3.6). If we use the unique geodesic joining $f(x)$ to $h(x)$ in the normal neighborhood $U(f(x), \delta(x))$ of $[17$, p. 165, (3.4)], a homotopy between $f$ and $h$ is constructed, so that $f_{*}=h_{*}$. As a result, we may as well suppose that $f=h$, i.e., that $f\left(B_{f}\right) \subset f\left(R_{n-2}(f)\right)$, so that [25, p. 173, Theorem 2] $\operatorname{dim}\left(f\left(B_{f}\right)\right) \leqq n-2$. Since $f$ is monotone, $B_{f}=f^{-1}\left(f\left(B_{f}\right)\right)$.

Let $\alpha \in \pi_{1}\left(M^{n}, x\right)$ with $f_{*}(\alpha)=0$. As above $\alpha$ has a representative $\mu: S^{1} \rightarrow M^{n}$ 
which is a polyhedral embedding, and we may suppose that $\mu\left(S^{1}\right) \cap B_{f}=\varnothing$. Let $D^{2}$ be the unit 2-disk with boundary $S^{1}$. Since $f \mu$ is homotopic to a constant map, there exists a map $F: D^{2} \rightarrow N^{n}$ such that the restriction $F \mid S^{1}=f \mu$. There exists a simplicial approximation $G$ to $F$ such that $G\left(S^{1}\right) \cap f\left(B_{f}\right)=\varnothing$ and $f^{-1} G \mid S^{1}$ is a representative of $\alpha$ also. Let $\xi$ be a $C^{3}$ diffeomorphism of $N^{n}$ onto itself such that $f$ is transverse regular on each (open) simplex of $\xi^{-1} G\left(D^{2}\right)$ [29, p. 26 and p. 27] (e.g., see the proof of [9, Lemma 3]); choose $\xi$ sufficiently near the identity that $f^{-1} \xi^{-1} G \mid S^{1}$ is again a representative of $\alpha$. Since $f\left(B_{f}\right) \subset f\left(R_{n-2}(f)\right)$, the 1-skeleton of $\xi^{-1}\left(G\left(D^{2}\right)\right)$ is disjoint from $f\left(B_{f}\right)$. For each closed 2-simplex $\tau$ of $\xi^{-1} G\left(D^{2}\right)$, $f^{-1}(\tau)$ is a (topological) embedding of a 2-manifold with boundary homeomorphic to $S^{1}\left[29\right.$, p. 23]. If $f^{-1}(\tau)$ is a 2-cell for each such 2-simplex $\tau$, then $\alpha=0$. Thus we may suppose that for some $\tau, f^{-1}(\tau)$ is not a closed 2-cell; we will obtain a contradiction.

There is a $C^{3}$ diffeomorph $E \subset N^{n}$ of $E^{2}$ such that $\tau \subset E$ and $f$ is transverse regular on $E$; we may suppose (3.2) that $E$ is a $C^{\infty}$ submanifold of $N^{n}$. Since bdy $\tau \cap f\left(B_{f}\right)$ $=\varnothing$, there is a $C^{\infty}$ embedding $\lambda$ of the closed unit disk $D^{2}$ into $E$ such that $\lambda\left(D^{2}\right) \subset$ int $\tau$ and $\left(\tau-\lambda\left(\right.\right.$ int $\left.\left.D^{2}\right)\right) \cap f\left(B_{f}\right)=\varnothing$; by [29, p. 26] we may suppose that $f$ is transverse regular on $\lambda\left(\partial D^{2}\right)$. Then $f^{-1}(\tau)$ is a 2-cell if and only if $f^{-1}\left(\lambda\left(D^{2}\right)\right)$ is also a 2-cell, so that we may as well suppose that $\tau=\lambda\left(D^{2}\right)$; let $J^{2}=f^{-1}(\tau)$.

Let $\Sigma$ be the diffeomorph of $S^{2}$ given by (3.5), and let $h$ be the $C^{3}$ map given by (3.4) for $f, K_{0}^{p}=\Sigma s=1$, and $K_{1}^{p}=\tau$. Let $g: J^{2} \times E^{n-2} \rightarrow D^{2} \times E^{n-2}$ be the restriction of $h$. Then $g$ is a $C^{3}$ monotone onto map, $g\left(J^{2} \times\{t\}\right)=D^{2} \times\{t\}$ for each $t \in E^{n-2}$, $g^{-1}\left(\partial D^{2} \times E^{n-2}\right)=\partial J^{2} \times E^{n-2}, n \geqq 3, \operatorname{dim}\left(B_{g}\right) \leqq n-2$, and (since $g$ is transverse regular on each $\partial D^{2} \times\{t\}$ and $\left.f\left(B_{f}\right) \subset f\left(R_{n-2}(f)\right)\right)$

$$
g\left(B_{g}\right) \subset g\left(R_{n-2}(g)\right) \cap\left(\operatorname{int}\left(D^{2}\right) \times E^{n-2}\right) .
$$

Let $g_{t}: J^{2} \times\{t\} \rightarrow D^{2} \times\{t\}$ be the restriction of $g$, and let $B\left(g_{t}\right)$ be its branch set. Since

$$
g\left(R_{n-2}(g)\right) \cap\left(\left(\text { int } D^{2}\right) \times\{t\}\right)=g_{t}\left(R_{0}\left(g_{t}\right)\right),
$$

$\operatorname{dim}\left(g_{t}\left(R_{0}\left(g_{t}\right)\right)\right) \leqq 0[25$, p. 173 , Theorem 2], and

$$
g_{t}\left(B\left(g_{t}\right)\right) \subset g\left(B_{g}\right) \cap\left(\left(\text { int } D^{2}\right) \times\{t\}\right)
$$

we have $\operatorname{dim}\left(g_{t}\left(B\left(g_{t}\right)\right)\right) \leqq 0$.

Suppose that there is a $t \in E^{n-2}$ such that, for each $y \in D^{2}, H^{1}\left(g^{-1}(y, t) ; Z_{2}\right)=0$. It follows from the Vietoris Mapping Theorem [1] (cf. (4.3)) applied to $g_{t}$ that $H^{1}\left(J^{2} ; Z_{2}\right)=0$; since $J^{2}$ is not a 2-cell, a contradiction results. Thus, for each $t \in E^{n-2}$ there exists at least one $y \in D^{2}$ such that $H^{1}\left(g^{-1}(y, t) ; Z_{2}\right) \neq 0$. By [8, p. $372,(2.3)]$ the number of such $y$ is at most $\operatorname{dim}\left(H^{1}\left(J^{2} ; Z_{2}\right)\right)$. A contradiction is now deduced as in the proof of [8, p. 372, (2.4), Second Case]. (Since $g$ is monotone, that proof can be simplified somewhat-in particular, paragraphs three and four can be omitted.) 
4.2. Lemma. Let $f$ be $C^{p+1}$ monotone with $\operatorname{dim}\left(B_{f}\right) \leqq n-2$, let $n \geqq 3$, and let $B^{p}$ be a $C^{p+1}$ diffeomorph of a p-ball in $N^{n}$ such that $f$ is transverse regular $[29, \mathrm{p} .23]$ on both $B^{p}$ and the boundary $\partial B^{p}$. Then $f^{-1}\left(B^{p}\right)$ and $f^{-1}\left(\partial B^{p}\right)$ are simply connected for $p \geqq 3$, and $f^{-1}\left(B^{2}\right)$ is a closed 2-cell.

Proof. The map $f$ is onto by $(2.2)(2)(\mathrm{i})$; by [32, p. 138, (2.2)] $f^{-1}\left(B^{p}\right)$ and $f^{-1}\left(\partial B^{p}\right)$ are connected (for $p \geqq 2$ ) manifolds [29, p. 23].

For $p<n$ let $\Sigma$ be the $C^{p+1}$ diffeomorph of $S^{p}$ given by (3.5), and let $h$ be the $C^{p+1}$ map given by (3.4) for $f, K_{0}^{p}=\Sigma$ and $K_{1}=\partial B^{p}$. Since $h^{-1}\left(\operatorname{int}\left(B^{p}\right) \times S(0, \varepsilon)\right)$ $=f^{-1}\left(\operatorname{int}\left(B^{p}\right)\right) \times S(0, \varepsilon)$, it follows from (4.1) that $\pi_{1}\left(f^{-1}\left(\operatorname{int}\left(B^{p}\right)\right)\right)=0$; also from (4.1) $\pi_{1}\left(f^{-1}\left(\operatorname{int}\left(B^{n}\right)\right)\right)=0$. Since $f^{-1}\left(\partial B^{p}\right)$ is collared in $f^{-1}\left(B^{p}\right)[22$, p. 51, (5.9)] (or [20, p. 23, (3.6)]), $\pi_{1}\left(f^{-1}\left(B^{p}\right)\right)=0$. Thus $f^{-1}\left(B^{2}\right)$ is a 2-cell.

For $p \geqq 3$ it similarly follows from application of (3.4) to $K_{0}=\partial B^{p}$ that $\pi_{1}\left(f^{-1}\left(\partial B^{p}\right)\right)=0$.

4.3. Remark. The Vietoris Mapping Theorem. If $X$ and $Y$ are compact metric spaces, and $f: X \rightarrow Y$ is acyclic (integral Čech cohomology), then $f$ induces an isomorphism $H^{j}(X ; Z) \approx H^{j}(Y ; Z)$.

To obtain this form of the theorem from that given in [1] use the duality [16, p. 141, (F)] between the Čech homology and cohomology groups $H_{j}\left(X ; R_{1}\right)$ and $H^{j}(X ; Z)$, where $R_{1}$ is the group of real numbers modulo 1 , and the fact that the modified Vietoris homology groups of [1] agree with the Čech homology groups $[1$, p. 536].

4.4. The proof of (1.1b). The hypothesis that $\operatorname{dim}\left(B_{f}\right) \leqq n-2$ is required (4.5). Given any compact set $X \subset S^{n}$ with $S^{n}-X C^{\infty}$ diffeomorphic to $E^{n}$, it follows from (2.4) that there is a $C^{\infty}$ monotone onto map $f: S^{n} \rightarrow S^{n}$ with $S^{n}-X$ mapped diffeomorphically onto $S^{n}-\{p\}$ and $f(X)=\{p\}$.

Proof. See (1.5); let $y \in N^{n}$, and let $U$ be a neighborhood of $f^{-1}(y)$ in $M^{n}$. Choose a $C^{\infty}$ diffeomorph $B^{n} \subset N^{n}$ of the closed $n$-ball $D^{n} \subset E^{n}$ such that $y \in \operatorname{int}\left(B^{n}\right)$ and $f^{-1}\left(B^{n}\right) \subset U$; by the Thom Transversality Theorem [29, p. 26] we may suppose that $f$ is transverse regular on $\partial B^{n}$. Thus $f^{-1}\left(\partial B^{n}\right)$ is a connected (by (4.3)) $C^{n}$ $(n-1)$-manifold [29, p. 23] which separates $U$, so that $f^{-1}\left(B^{n}\right)$ is a $C^{n} n$-manifold with boundary. By (4.3) $f^{-1}\left(B^{n}\right)$ is acyclic, and thus by (4.2) and the Hurewicz Theorem is a homotopy cell. If $n \neq 3,4,5$, it follows from the $h$-cobordism theorem $\left[20\right.$, p. 108] that $f^{-1}\left(B^{n}\right)$ is $C^{n}$ diffeomorphic to the closed $n$-ball $D^{n}$. Since $y$ and $U$ were arbitrary, $f$ has the desired property.

4.5. ExAMPLE. An acyclic (integral Čech cohomology) $C^{\infty}$ map need not be cellular. Let $K^{3}$ be a polyhedral homology 3-sphere [26, pp. 216-218]; there is [5, p. 797] a $C^{\infty}$ manifold $M^{3}$ homeomorphic with $K^{3}$. By an elementary argument there exists a 2-dimensional subpolyhedron $X$ such that $M^{3}-X$ is homeomorphic to $E^{3}$ (for a more general result in this direction see [11] and [2]); by [23, p. 544, (6.3)] $M^{3}-X$ is $C^{\infty}$ diffeomorphic to $E^{3}$. Define $f: M^{3} \rightarrow S^{3}$ by: $f(X)$ is a single point $p$, and $f$ maps $M^{3}-X C^{\infty}$ diffeomorphically onto $S^{3}-\{p\}$; by (2.4) we may suppose that $f$ is $C^{\infty}$. 
Since $0=H^{2}\left(S^{3} ; Z\right) \approx H^{2}\left(S^{3}, f(X) ; Z\right) \approx H^{2}\left(M^{3}, X ; Z\right)[12$, p. 266], it follows from the cohomology sequence that $H^{1}(X ; Z)=0$. From (2.12) (or again from the cohomology sequence), $H^{2}(X ; Z)=0$. Thus $f$ is acyclic.

If $f$ is cellular, then there is a topological (closed) 3-cell $A \subset M^{3}$ such that $X \subset$ int $A$; there is a bicollared [4, p. 85] 3-cell $B \subset$ int $A$ with $X \subset$ int $B$. From [3] $M^{3}-$ int $A\left(=E^{3}-\right.$ int $\left.A\right)$ is a closed 3-cell, and thus $M^{3}$ is homeomorphic to $S^{3}$, contradicting our assumption. Hence $f$ is not cellular.

4.6. The proof of (1.1a). If $n=1$ or 2 , then $f$ is a homeomorphism; thus we may suppose that $n \geqq 3$. We suppose that $f$ is not acyclic, and will obtain a contradiction. Then (1.1b) yields (1.1a).

There is a minimal integer $p(p=0,1, \ldots, n)$ such that there are (i) a $C^{n}$ submanifold $\Gamma^{p} \subset N^{n}\left(\partial \Gamma^{p}=\varnothing\right)$ on which $f$ is transverse regular, and (ii) a point $y_{1} \in \Gamma^{p}$ with $f^{-1}\left(y_{1}\right)$ not acyclic. By (2.2)(2i) $f$ is monotone onto, and by (2.2)(iii) $p \geqq 2$. The set $f^{-1}\left(y_{1}\right)$ is the nested intersection of sets $f^{-1}\left(B_{j}^{p}\right)(j=1,2, \ldots)$, where each $B_{j}^{p}$ is a $C^{n}$ diffeomorph of a $p$-ball in $\Gamma^{p}$ and $f$ is transverse regular on $\partial B_{j}^{p}$. By the Continuity Theorem [12, p. 261] there exist $j$ and $i(i=1,2, \ldots, p-1)$ with $H^{i}\left(f^{-1}\left(B_{j}^{p}\right) ; Z\right) \neq 0$; let $B_{j}^{p}$ be denoted by $B^{p}$. By (4.2) $p \geqq 3$. From the definition of $p$ the restriction map $f \mid f^{-1}\left(\partial B^{p}\right): f^{-1}\left(\partial B^{p}\right) \rightarrow \partial B^{p}$ is acyclic, so that by the Vietoris Mapping Theorem (4.3) $\partial B^{p}$ is a cohomology sphere.

By (4.2) $\pi_{1}\left(f^{-1}\left(B^{p}\right)\right)=0$, so that $f^{-1}\left(B^{p}\right)$ is orientable. Suppose that

$$
H^{i}\left(f^{-1}\left(B^{p}\right) ; Z\right)=0
$$

for every $i \geqq p / 2$. By the Universal Coefficient Theorem [19, p. 172, Example 2] the same is true for every coefficient field $F$. From the Lefschetz Duality Theorem $H^{i}\left(f^{-1}\left(B^{p}\right), f^{-1}\left(\partial B^{p}\right) ; F\right)=0$ for every $i \leqq p / 2$, and from the cohomology sequence and the fact that $H^{j}\left(f^{-1}\left(\partial B^{p}\right) ; F\right)=0$ for $j<p-1$, it follows that $H^{i}\left(f^{-1}\left(B^{p}\right) ; F\right)=0$ for all $i$; thus $H^{i}\left(f^{-1}\left(B^{p}\right) ; Z\right)=0$ for all $i$. As a result we may suppose that

$$
\text { for some } i \geqq p / 2, \quad H^{i}\left(f^{-1}\left(B^{p}\right) ; Z\right) \neq 0 .
$$

Let $\Delta^{p, q}$ be the $q$ th barycentric subdivision of the closed $p$-simplex $\Delta^{p}$. Given any $\delta>0$, there are $q \geqq 1$ and a $C^{n}$ triangulation [22, pp. 76-77] $\gamma$ mapping $\Delta^{p, q}$ onto $B^{p}$ with mesh at most $\delta$ (e.g., see [23, p. 546]); by applications of [29, p. 26] (cf. the proof of [9, Lemma 3]) we may suppose that $f$ is transverse regular on each open simplex $\gamma(\sigma)$ of each dimension, so that $f^{-1}(\gamma(\sigma))$ is a $C^{n}$ manifold. From the definition of $p$ and the Vietoris Mapping Theorem (4.3), $f^{-1}(\gamma(\sigma))$ is acyclic for each closed simplex $\sigma$ with $\operatorname{dim} \sigma<p$. Now from the Mayer-Vietoris sequence $H^{j}\left(f^{-1}\left(B^{p}\right) ; Z\right) \approx \sum_{\tau} H^{j}\left(f^{-1}(\gamma(\tau)) ; Z\right)$, direct sum over the closed $p$-simplices $\tau$ of $\Delta^{p, q}$; thus $f^{-1}(\gamma(\tau))$ is acyclic for all but at most $m$ closed $p$-simplices $\tau$, where $m$ is the minimal number of generators of $H^{*}\left(f^{-1}\left(B^{p}\right) ; Z\right)$.

Given any $\eta>0$ and any closed $p$-simplex $\tau$ of $\Delta^{p, q}, \gamma(\tau)$ has an analogous subdivision of mesh at most $\eta$, and it follows from the Continuity Theorem [12, p. 261] that 
(2) $f^{-1}(y)$ is acyclic for all but at most $m$ points $y \in \operatorname{int}\left(B^{p}\right)$, and

$$
H^{*}\left(f^{-1}\left(B^{p}\right) ; Z\right) \approx \sum_{y \in \operatorname{int}\left(B^{p}\right)} H^{*}\left(f^{-1}(y) ; Z\right) .
$$

By (1) there is a point $x \in \operatorname{int}\left(B^{p}\right)$ with $H^{i}\left(f^{-1}(x) ; Z\right) \neq 0$; since $f^{-1}(x) \subset B_{f}$, $\operatorname{dim}\left(B_{f}\right) \geqq i \geqq p / 2[16, \mathrm{p} .137$, (F)]. In case $p=n$ a contradiction results, and thus $p=3,4, \ldots$, or $n-1$.

Let $\Sigma$ be the $C^{n}$ diffeomorph of $S^{p}$ given by (3.5) with $B^{p} \subset \Sigma \subset N^{n}$, and let $h=\rho f \omega$ be the $C^{n}$ map given by (3.4) for $f, K_{0}^{p}=\Sigma$, and $K_{1}^{p}=\partial B^{p}$; then $h$ maps $f^{-1}\left(B^{p}\right) \times E^{n-p}$ onto $B^{p} \times E^{n-p}$ with $h\left(f^{-1}\left(B^{p}\right) \times\{t\}\right)=B^{p} \times\{t\}$ and $h$ transverse regular on $\partial B^{p} \times\{t\}$ for each $t \in E^{n-p}$. By the preceding argument, for each $t \in E^{n-p}$ there are at most $m$ points $y \in \operatorname{int}\left(B^{p}\right)$ such that $h^{-1}(y, t)$ is not acyclic. Thus there is a $t$ such that the number of points $y \in \operatorname{int}\left(B^{p}\right)$ with $h^{-1}(y, t)$ not acyclic is maximal. We may as well suppose that $t=0$.

Let $y_{j}(j=1,2, \ldots, s)$ be these points in $\operatorname{int}\left(B^{p}\right)$, and let $B_{j}^{p}$ be $C^{n}$ diffeomorphs of a $p$-ball in int $\left(B^{p}\right)$ such that the sets $B_{j}^{p}$ are mutually disjoint, $y_{j} \in \operatorname{int}\left(B_{j}^{p}\right)$, and $h$ is transverse regular on $\partial B_{j}^{p} \times\{0\}$. By (2) applied to $B_{j}^{p}, H^{*}\left(f^{-1}\left(B_{j}^{p} ; Z\right)\right) \neq 0$. Let $\chi$ be the $C^{n}$ diffeomorphism given by (3.4) applied to $h$ itself, with $\rho$ the identity map, $K_{0}^{p}=\Sigma \times\{0\}, K_{j}^{p}=\partial B_{j}^{p} \times\{0\}(j=1,2, \ldots, s)$, and $K_{s+1}^{p}=\partial B^{p} \times\{0\}$. For each $j$ $(j=1,2, \ldots, s)$ and $t \in S(0, \varepsilon),(h \chi)^{-1}\left(B_{j}^{p} \times\{t\}\right) \approx f^{-1}\left(B_{j}^{p}\right)$, and so by (2) there is at least one point $y(j, t) \in \operatorname{int}\left(B_{j}^{p}\right)$ with $(h \chi)^{-1}((y(j, t), t))$ not acyclic. By the maximality choice there is exactly one such point.

Thus for each $t \in S(0, \varepsilon)$ there is exactly one point $\alpha(t) \in \operatorname{int}\left(B_{1}^{p}\right) \times\{t\}$ with $(h \chi)^{-1}(\alpha(t))$ not acyclic; by (1) and (2) $H^{i}\left((h \chi)^{-1}(\alpha(t)) ; Z\right) \neq 0$ for some $i \geqq p / 2$.

The proof now parallels (but differs from) the last half of Case 2 in the proof of $[8$, p. $372,(2.4)]$. We next prove that the one-to-one function $\alpha: S(0, \varepsilon) \rightarrow \operatorname{int}\left(B_{1}^{p}\right)$ $\times S(0, \varepsilon)$ is continuous; suppose the contrary. Then there exist $t_{r} \in S(0, \varepsilon)(r=1$, $2, \ldots)$ with $t_{r} \rightarrow t_{0}, \alpha\left(t_{r}\right) \rightarrow z, z \neq \alpha\left(t_{0}\right)$. Choose a $C^{n}$ diffeomorph $A^{p} \subset \Sigma$ of the unit $p$-ball with $z \in \operatorname{int}\left(A^{p}\right) \times\left\{t_{0}\right\}, \alpha\left(t_{0}\right) \notin A^{p} \times\left\{t_{0}\right\}$, and $h_{\chi}$ transverse regular on $\partial A^{p} \times\left\{t_{0}\right\}$. There exists $\zeta>0$ such that $h \chi$ is transverse regular on $\partial A^{p} \times\{t\}$ for each $t \in S\left(t_{0}, \zeta\right)$; let $\xi$ be the diffeomorphism given by (3.4) for the restriction map $h_{\chi} \mid\left(\Sigma \times S\left(t_{0}, \zeta\right)\right), \rho$ the identity map, $K_{0}=\Sigma \times\left\{t_{0}\right\}$, and $K_{1}=\partial A^{p} \times\left\{t_{0}\right\}$. If $A^{p} \times\left\{t_{0}\right\}$ is identified with $A^{p}$, then $h_{\chi} \xi$ maps $\left(h_{\chi}\right)^{-1}\left(A^{p}\right) \times\{t\}$ onto $A^{p} \times\{t\}$ for each $t$ in some neighborhood of $t_{0}$. Since $\alpha\left(t_{0}\right) \notin A^{p} \times\left\{t_{0}\right\},(h \chi)^{-1}\left(A^{p}\right)$ is acyclic by (2); since $\alpha\left(t_{r}\right) \in \operatorname{int}\left(A^{p}\right) \times\left\{t_{r}\right\}$ for $r$ sufficiently large, $\left(h_{\chi}\right)^{-1}\left(A^{p}\right)$ is not acyclic by (2). From this contradiction it follows that $\alpha$ is continuous, and thus a homeomorphism into.

We may suppose that $B_{1}^{p}$ is the closed unit $p$-ball $D^{p}$; let $e: D^{p} \times S(0, \varepsilon)$ $\rightarrow D^{p} \times S(0, \varepsilon)$ be the restriction of $h \chi$, and let $\pi: D^{p} \times S(0, \varepsilon) \rightarrow D^{p}$ be projection. Since $\alpha(t) \in \operatorname{int}\left(D^{p}\right) \times\{t\}$, we may suppose (by replacing $\varepsilon$ by a smaller number if necessary) that there exists $\eta>0$ with $|x-\pi \alpha(t)|>\eta$ for all $x \in \partial D^{p}$ and $t \in S(0, \varepsilon)$. For each $\phi, 0 \leqq \phi \leqq \eta$, let

$$
A_{\phi}=\{(x, t):|x-\pi \alpha(t)| \leqq \phi \quad \text { and } \quad t \in S(0, \varepsilon)\} .
$$


If $B(0, \phi)$ is the closed ball of radius $\phi$ and center 0 in $E^{p}$, the function $\beta: B(0, \phi)$ $\times S(0, \varepsilon) \rightarrow A_{\phi}$ defined by $\beta(x, t)=(x+\pi \alpha(t), t)$ is a homeomorphism. Each $A_{\phi} \cap\left(D^{p} \times\{t\}\right)$ is a (geometric) $p$-ball in $\operatorname{int}\left(D^{p}\right) \times\{t\}$, so there is a canonical deformation retraction of $D^{p} \times\{t\}$ onto $A_{\phi} \cap\left(D^{p} \times\{t\}\right)$, retracting along radial lines from $\pi \alpha(t)$; these retractions define a deformation retraction of $D^{p} \times S(0, \varepsilon)$ onto $A_{\phi}$.

Fix $\phi>0$, and let $U$ and $V$ be the one point compactifications of $\left(D^{p} \times S(0, \varepsilon)\right)$ $-\operatorname{int}\left(A_{\phi}\right)$ and $\partial A_{\phi}$, respectively, with added point $u$. Let $P, Q, R, S$, and $T$ be the one point compactifications of $e^{-1}\left(D^{p} \times S(0, \varepsilon)\right), \quad e^{-1}(\alpha(S(0, \varepsilon))), e^{-1}\left(A_{\phi}\right)$, $e^{-1}(U-\{u\})$, and $e^{-1}(V-\{u\})$, respectively, with added point $q$.

Now

$$
H^{k}(P ; Z) \approx H^{k}(P,\{q\} ; Z) \quad(k=1,2, \ldots) .
$$

Since $e^{-1}\left(D^{p} \times S(0, \varepsilon)\right)$ is homeomorphic to $f^{-1}\left(B_{1}^{p}\right) \times E^{n-p}, H^{k}(P,\{q\} ; Z)$ is isomorphic to $H^{k}\left(f^{-1}\left(B_{1}^{p}\right) \times S^{n-p}, f^{-1}\left(B_{1}^{p}\right) \times\{z\} ; Z\right)[12$, p. 266], where $z$ is any point of $S^{n-p}$. We may suppose that $i$ is the largest integer for which $H^{i}\left(f^{-1}\left(B_{1}^{p}\right) ; Z\right)$ $\neq 0$, so that $H^{n-p+i}\left(f^{-1}\left(B_{1}^{p}\right) ; Z\right)=0$. From the exactness of the cohomology sequence

$$
\psi^{*}: H^{n-p+i}\left(f^{-1}\left(B_{1}^{p}\right) \times S^{n-p}, f^{-1}\left(B_{1}^{p}\right) \times\{z\} ; Z\right) \rightarrow H^{n-p+i}\left(f^{-1}\left(B_{1}^{p}\right) \times S^{n-p} ; Z\right)
$$

is an epimorphism ( $\psi$ is inclusion), and by the Künneth Formula the latter group is isomorphic to $H^{i}\left(f^{-1}\left(B_{1}^{p}\right) ; Z\right)$. Thus $H^{n-p+i}(P ; Z) \neq 0$.

Since $V$ is a deformation retract of $U, H^{j}(U ; Z) \approx H^{j}(V ; Z)$. From the Vietoris Mapping Theorem (3.4) $H^{j}(U ; Z) \approx H^{j}(S ; Z)$ and $H^{j}(V ; Z) \approx H^{j}(T ; Z)$, and hence the inclusion map induces an isomorphism $H^{j}(S ; Z) \approx H^{j}(T ; Z)$. As a result $H^{j}(S, T ; Z)=0$, and, by excision [12, p. 266], $H^{j}(P, R ; Z)=0$. Thus inclusion induces an isomorphism $H^{j}(P ; Z) \approx H^{j}(R ; Z)$. Since $\phi$ may be chosen arbitrarily small, it follows from the Continuity Theorem $\left[12\right.$, p. 261] that $H^{j}(P ; Z)$ $\approx H^{j}(Q ; Z)$. Thus $H^{n-p+i}(Q ; Z) \neq 0$.

From [16, p. 137, (F)] $\operatorname{dim}(Q-\{q\}) \geqq n-p+i$. By the choice of $Q, H^{i}\left(e^{-1}(e(x)) ; Z\right)$ $\neq 0(i>0)$ and $e^{-1}(e(x)) \subset Q$ for each $x \in Q-\{q\}$, so that $Q-\{q\} \subset B_{e}$. Since the branch set $B_{e}$ is homeomorphic to a subset of $B_{f}, \operatorname{dim}\left(B_{f}\right) \geqq n-p+i$. Since $p / 2 \leqq i$ and $p<n, n-p+i>n / 2$, contradicting the hypothesis on $\operatorname{dim}\left(B_{f}\right)$.

4.7. REMARK. Under the hypotheses of (1.1a) or (1.1b), if $M^{n}$ and $N^{n}$ are compact and simply connected, then $f$ is a homotopy equivalence.

Proof. Since $f$ is acyclic, it induces (4.3) isomorphisms $f^{*}: H^{i}\left(M^{n} ; Z\right)$ $\rightarrow H^{i}\left(N^{n} ; Z\right)$ for all $i$. Because $M^{n}$ and $N^{n}$ are finite polyhedra [22, p. 101], $f$ thus induces isomorphisms $f_{*}: H_{i}\left(M^{n} ; Z\right) \rightarrow H_{i}\left(N^{n} ; Z\right)$ for all $i$ (use [19, p. 172, Example 2] to prove the dual of [19, p. 81, Corollary 4.6]). The remark follows from [13, p. 113, (3.8)].

4.8. REMARK. Let $M^{n}$ and $N^{n}$ be triangulated manifolds, and let $f: M^{n} \rightarrow N^{n}$ be simplicial and proper with $\operatorname{dim}\left(B_{f}\right) \leqq n-1$. 
(a) If $f$ is monotone onto, then $f$ is a homeomorphism.

(b) If $\operatorname{dim}\left(B_{f}\right) \leqq n-3$, then $f$ is a finite-to-one covering map.

Thus the analogs of (1.1), (1.2), and [8, p. 370, (1.1)] for simplicial maps are a fortiori true.

Proof. Since $\operatorname{dim}\left(B_{f}\right) \leqq n-1, f$ maps each simplex $\sigma$ of $M^{n}$ homeomorphically onto $f(\sigma)$; thus $f$ is light and $\operatorname{dim}\left(f\left(B_{f}\right)\right)=\operatorname{dim}\left(B_{f}\right)$. If $\operatorname{dim}\left(B_{f}\right)=n-1$, then there are two $n$-simplices $\sigma$ and $\tau$ with common $(n-1)$-face such that $f(\sigma)=f(\tau)$; thus, if $f$ is monotone, $\operatorname{dim}\left(B_{f}\right) \leqq n-2$. From $[10$, p. 608, (1.2)] it follows that if $f$ is monotone, then $B_{f}=\varnothing$, so that $f$ is a homeomorphism. In case (b) it similarly follows from $[10$, p. $608,(1.2)]$ that $B_{f}=\varnothing$, and since $f$ is proper, $f$ is a finite-to-one covering map.

5. Answer to a question of Hopf. In [14] H. Hopf asked the following question: If $f: S^{3} \rightarrow S^{2}$ is essential, is it true that each $y$ in $S^{2}$ has $\operatorname{dim}\left(f^{-1}(y)\right) \geqq 1[14, \mathrm{p}$. 284, (b)]? In fact, is the first Betti number positive (d)? An affirmative answer to the first question and (essentially) to the second is shown below.

5.1. TheOREM. If $f: S^{3} \rightarrow S^{2}$ is an essential map, then, for every $y \in S^{2}$, $H^{1}\left(f^{-1}(y) ; Z\right)$ has an element of infinite order. In particular $\operatorname{dim}\left(f^{-1}(y)\right) \geqq 1$.

Proof. By $\left[15\right.$, p. 68, (6.3) and (6.4)] $f=p F$, where $F: S^{3} \rightarrow S^{3}$ is essential and $p$ is the Hopf map $p: S^{3} \rightarrow S^{2}$. Given $y \in S^{2}$, let $D$ be a topological closed disk in $S^{2}$ such that $y \in$ int $D$, and let $\rho: D \times S^{1} \rightarrow D$ and $\sigma: D \times S^{1} \rightarrow S^{1}$ be the projection maps. Since $p$ is a bundle map, there exists a homeomorphism $h$ of $p^{-1}(D)$ onto $D \times S^{1}$ such that $\rho h=p$. We may suppose that $y$ is the origin 0 of the plane, that $D_{t}$ is the closed disk of radius $t$ about $0(0 \leqq t \leqq 1)$, and that $D=D_{1}$.

Suppose that the (restriction) map $\sigma h F \mid f^{-1}\left(D_{t}\right): f^{-1}\left(D_{t}\right) \rightarrow S^{1}$ is inessential for some $t, 0<t \leqq 1$; we may suppose that $t=1$. Let $G: f^{-1}\left(D_{1}\right) \times[0,1] \rightarrow S^{1}$ be the homotopy, where $G(x, 1)=\sigma h F(x)$ and $G(x, 0)=q \in S^{1}$. Define $H: f^{-1}\left(D_{1}\right)$ $\times[0,1] \rightarrow D_{1} \times S^{1} \quad$ by $H(x, u)=(f(x), G(x, u)) ; \quad$ then $H(x, 1)=h F(x)$ and $H\left(f^{-1}\left(D_{1}\right), 0\right)=D_{1} \times\{q\}$. Define maps $F_{s}: S^{3} \rightarrow S^{3}(0 \leqq s \leqq 1)$ by: $F_{s}=F$ off $f^{-1}\left(D_{1}\right)$; and for $x \in f^{-1}\left(\operatorname{bdy}\left(D_{t}\right)\right), F_{s}(x)=h^{-1} H(x, s+(1-s) t)$. Then $F_{1}=F, F$ is homotopic to $F_{0}$, and only one point of $p^{-1}(0)$ is in the range of $F_{0}$; hence $F$ is inessential, contradicting the hypothesis.

As a result $\sigma h F \mid f^{-1}\left(D_{t}\right)$ is essential $(0<t \leqq 1)$. It follows that the Brushlinsky group $\pi^{1}\left(f^{-1}\left(D_{t}\right)\right) \neq 0[15$, p. 47]; moreover the diagram

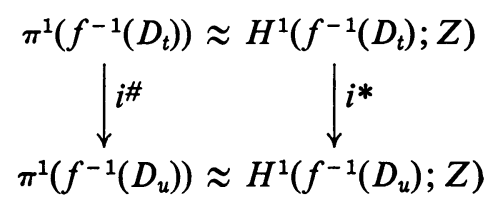

(where $t>u>0$, and $i^{*}$ and $i^{\#}$ are induced by inclusion) commutes [15, pp. 49, 59, (C)]. It follows from [12, p. 221, (4.4)] and the Continuity Theorem [12, p. 261] that $H^{1}\left(f^{-1}(y) ; Z\right) \neq 0$; thus $\operatorname{dim}\left(f^{-1}(y)\right) \geqq 1[16$, p. 137, (F)]. From the Universal 
Coefficient Theorem (and the Continuity Theorem), $H^{1}\left(f^{-1}(y) ; Z\right)$ has an element of infinite order.

5.2. Remarks. More generally, if $S^{3}$ is replaced by any finite polyhedron (e.g., a compact 3-manifold) and $f$ is algebraically trivial [15, p. 67], the same proof yields the conclusion. As Hopf points out, the strict analog of this theorem for higher dimensions is false: define $f: S^{4} \rightarrow S^{3}$ by suspension of $\mathrm{p}: S^{3} \rightarrow S^{2}$; there are two points $y_{i}$ for which $f^{-1}\left(y_{i}\right)$ is a single point.

The author is grateful to the referee for suggesting improvements in the presentation.

\section{REFERENCES}

1. E. G. Begle, The Vietoris mapping theorem for bicompact spaces, Ann. of Math. 51 (1950), 534-543.

2. M. Brown, "A mapping theorem for untriangulated manifolds" in Topology of 3-manifolds, edited by M. K. Fort, Jr., Prentice-Hall, Englewood Cliffs, N. J., 1962, 92-94.

3. - A proof of the generalized Schoenflies theorem, Bull. Amer. Math. Soc. 66 (1960), 74-76.

4. - "Locally flat embeddings of topological manifolds" in Topology of 3-manifolds, edited by M. K. Fort, Jr., Prentice-Hall, Englewood Cliffs, N. J., 1962, 83-91.

5. S. S. Cairns, Homeomorphisms between topological manifolds and analytic manifolds, Ann. of Math. 41 (1940), 796-808.

6. P. T. Church, Differentiable maps with non-negative Jacobian, J. Math. Mech. 16 (1966), 703-708.

7. - Differentiable open maps on manifolds, Trans. Amer. Math. Soc. 109 (1963), 87-100.

8. - Factorization of differentiable maps with branch set dimension at most $n-3$, Trans. Amer. Math. Soc. 115 (1965), 370-387.

9. - On points of Jacobian rank $k$. II, Proc. Amer. Math. Soc. 16 (1965), 1035-1038.

10. P. T. Church and E. Hemmingsen, Light open maps on n-manifolds. II, Duke Math. J. 28 (1961), 607-624.

11. P. H. Doyle and J. G. Hocking, A decomposition theorem for n-dimensional manifolds, Proc. Amer. Math. Soc. 13 (1962), 469-471.

12. S. Eilenberg and N. Steenrod, Foundations of algebraic topology, Princeton Univ. Press, Princeton, N. J., 1952.

13. P. J. Hilton, An introduction to homotopy theory, Cambridge Univ. Press, Cambridge, 1953.

14. H. Hopf, Uber den Defekt stetiger Abbildungen von Mannigfaltigkeiten, Rend. Mat. e Appl. (5) 21 (1962), 273-285.

15. S. T. Hu, Homotopy theory, Academic Press, New York, 1959.

16. W. Hurewicz and H. Wallman, Dimension theory, Princeton Univ. Press, Princeton. N. J., 1941.

17. S. Kobayashi and K. Nomizu, Foundations of differential geometry, Interscience, New York, 1963.

18. K. W. Kwun and F. Raymond, Almost acyclic maps of manifolds, Amer. J. Math. 86 (1964), 638-649.

19. S. MacLane, Homology, Academic Press, New York, 1963.

20. J. Milnor, Lectures on the h-cobordism theorem, Princeton Univ. Press, Princeton, N. J., 1965. 
21. - Morse theory, Princeton Univ. Press, Princeton, N. J., 1963.

22. J. R. Munkres, Elementary differential topology, Princeton Univ. Press, Princeton, N. J., 1963.

23. - Obstructions to the smoothing of piecewise-differentiable homeomorphisms, Ann. of Math. 72 (1960), 521-554.

24. R. S. Palais, Extending diffeomorphisms, Proc. Amer. Math. Soc. 11 (1960), 274-277.

25. A. Sard, Hausdorff measure of critical images on Banach manifolds, Amer. J. Math. 87 (1965), 158-174.

26. H. Seifert and W. Threlfall, Lehrbuch der Topologie, Chelsea, New York, 1947.

27. N. Steenrod, Topology of fiber bundles, Princeton Univ. Press, Princeton, N. J., 1951.

28. S. Sternberg, Lectures on differential geometry, Prentice-Hall, Englewood Cliffs, N. J., 1964.

29. R. Thom, Quelques propriétés globales des variétés différentiables, Comment. Math. Helv. 28 (1954), 17-86.

30. C. J. Titus, Sufficient conditions that a mapping be open, Proc. Amer. Math. Soc. 10 (1959), 970-973.

31. C. J. Titus and G. S. Young, A Jacobian condition for interiority, Michigan Math. J. 1 (1952), 89-94.

32. G. T. Whyburn, Analytic topology, Colloq. Publ., Vol. 28, Amer. Math. Soc., Providence, R. I., 1942.

33. — Topological analysis, Princeton Univ. Press, Princeton, N. J., 1958.

34. W. P. Ziemer, The structure of quasi-open maps, Duke Math. J. 32 (1965), 661-672.

SYRACUSE UNIVERSITY

Syracuse, New York 